\title{
The Nature of High-Redshift Galaxies
}

\author{
Rachel S. Somerville ${ }^{1,2}$, Joel R. Primack ${ }^{2} \&$ S. M. Faber ${ }^{3}$ \\ ${ }^{1}$ Racah Institute of Physics, The Hebrew University, Jerusalem \\ ${ }^{2}$ Department of Physics, University of California, Santa Cruz \\ ${ }^{3}$ UCO/Lick Observatory, University of California, Santa Cruz
}

4 September 2018

\begin{abstract}
Using semi-analytic models of galaxy formation, we investigate the properties of high redshift galaxies $(z \sim 3)$ and compare them with the observed population of Lymanbreak galaxies (LBGs). In addition to the usual quiescent mode of star formation, we introduce a physical model for starbursts triggered by galaxy-galaxy interactions. We find that with the merger rate that arises naturally in the merging hierarchy in CDM-type models, a significant fraction of bright galaxies identified at high redshift $(z \gtrsim 2)$ may be low-mass, bursting satellite galaxies within massive dark matter halos. The abundance of bright galaxies as a function of redshift and the luminosity function at $z \sim 3$ both appear to be in better agreement with the data when the starburst mode is included, especially when the effects of dust are considered. The objects that we identify as "LBGs" have star formation rates, half-light radii, I-K colours, and internal velocity dispersions that are in good agreement with the available data. We also investigate global quantities such as the star formation rate density and cold gas and metal content of the Universe as a function of redshift. Based on the combined constraints from these observations, we propose a scenario in which quiescent star formation at $z \gtrsim 2$ is relatively inefficient, and most of the observed LBGs are starbursts. We argue that this "bursting satellite" scenario can resolve the apparent paradox of LBGs that cluster like massive dark matter halos but have narrow line-widths.
\end{abstract}

Key words: galaxies: formation - galaxies: evolution - galaxies: starburst - cosmology: theory

\section{INTRODUCTION}

With the dramatic recent advances in observational astronomy, more and more pieces of the puzzle of galaxy formation and evolution are becoming available. Some of the important puzzle pieces include the number densities, colours, sizes, morph logies, internal velocity dispersions and star formation rates of bright star forming galaxies spanning a redshift range from $z \sim 0$ to $z \sim 4$, and the complementary information on the neutral hydrogen and metal content of the Universe to $z \sim 4$ obtained from quasar absorption systems. However, it still remains to fit these pieces together into a comprehensive and compelling theoretical framework.

Our window onto the high redshift Universe $(z \gtrsim 2)$ has been expanded tremendously by the "Lyman-break" photometric selection technique developed by Steidel and collaborators (Steidel \& Hamilton 1992; Steidel \& Hamilton 1993; Steidel et al. 1996a). This technique uses specially developed filters to exploit the redshifted Lyman-limit discontinuity in order to identify high-redshift candidates $(\bar{z} \sim 3)$. Similar techniques were exploited by Madau et al. (1996) to identify high-redshift candidates in the Hubble Deep Field (HDF; Williams et al. 1996). Extensive spectroscopic followup work at the Keck telescope has verified the accuracy of the photometric selection technique Steidel et al. 1996b; Lowenthal et al. 1997). The morphologies and sizes of these objects can be studied using the HDF sample Giavalisco, Steidel, \& Macchetto 1996; Lowenthal et al. 1997), and their clustering properties can be studied using the growing sample of hundreds of Lyman-break galaxies with spectroscopically confirmed redshifts (Steidel et al. 1998; Giavalisco et al. 1998; Adelberger et al. 1998).

One interpretation of the Lyman-break galaxies (LBGs), proposed by Steidel et al. (1996a) and reiterated by Giavalisco, Steidel, \& Macchetto (1996) and Steidel et al. (1998), is that they are located in the centers of massive dark matter halos $\left(M \gtrsim 10^{12} M_{\odot}\right)$ and have been forming stars at a moderate rate over a fairly long time-scale $(\gtrsim 1$ Gyr). This scenario supposes that the galaxies identified as LBGs at $z \sim 3$ are the direct progenitors of today's massive, luminous ellipticals and spheroids. The massive progenitor 
scenario has been supported by the semi-analytic modelling work of Baugh et al. (1998, hereafter BCFL98).

In this paper we investigate the viability of an alternative interpretation of the observations. There is clear observational evidence that galaxy-galaxy interactions trigger "starbursts", a mode of star formation with a sharply increased efficiency over a relatively short time-scale. There is also observational evidence that galaxy-galaxy interactions and starbursts are more common at high redshift than they are today (cf. Guzman et al. 1997). The similarity between the appearance of the spectra of the LBGs and local starburst galaxies has often been noted (Lowenthal et al. 1997 ; Pettini et al. 1997b). It seems plausible that at least some of the observed Lyman-break galaxies are relatively low-mass $\left(\sim 10^{9}-10^{10} M_{\odot}\right)$ objects in the process of an intense starburst. If a significant fraction of the objects are of this nature, this would have far-reaching implications for the interpretation of the observations.

Semi-analytic models are the best available means of performing an investigative exploration of the viability of this alternative scenario. Current numerical simulations cannot simultaneously resolve the small-mass objects that we are interested in and treat a cosmological volume, and there is still much uncertainty about how to treat the physical processes of gas dynamics, star formation, supernovae feedback, etc., that are certain to be important ingredients in the formation of these objects. Describing these processes requires several parameters, and only with semi-analytic models can the relevant parameter space be explored. In this paper, we will investigate whether starburst galaxies are expected to comprise a substantial fraction of the population of bright galaxies identified at high redshift, and whether these objects have properties that are compatible with the observed Lyman-break galaxies. In addition, we will investigate whether this scenario leads to self-consistent agreement with observations of global quantities such as the star formation rate density and the cold gas and metal content of the Universe as a function of redshift.

The outline of the paper is as follows. In Section 2.1, we give a brief introduction to the basic semi-analytic models. In Section 2.2, we present our simple approach for including merger-induced starbursts in our models. In Section B, we present the model predictions for the abundance of "LBGs" from $2 \lesssim z \lesssim 6$ and their properties at $z \sim 3$, such as the luminosity function, star-formation rates, ages, colours, and internal velocity dispersions. In Section 1 , we show how our results depend on various assumptions, such as the underlying cosmology, IMF, stellar metallicity of the model SEDs, and the recipe used for quiescent star formation. We show the predictions of our models for the evolution of global quantities such as the star formation rate density and cold gas and metal content of the Universe in Section . We conclude in Section 6.

\section{SEMI-ANALYTIC MODELLING}

\subsection{The Basic Models}

Semi-analytic techniques allow one to model the formation and evolution of galaxies in a hierarchical framework, including the effects of gravitation in the formation and merging of dark matter halos, and the hydrodynamics of gas cooling, star formation, supernovae feedback and metal production, galaxy-galaxy merging, and the evolution of stellar populations. The semi-analytic models used here are described in detail in Somerville (1997) and Somerville \& Primack (1998, hereafter SP98). As we showed in SP98, these models are in reasonably good agreement with a broad range of local galaxy observations, including the Tully-Fisher relation, the B-band luminosity function, cold gas contents, metallicities, and colours. Our basic approach is very similar in spirit to the models originally presented by Kauffmann, White, \& Guiderdoni (1993, hereafter KWG93) and Cole et al. (1994, hereafter CAFNZ94), and subsequently developed by these groups in numerous other papers. We refer the reader to SP98 for a more comprehensive review of the literature and of the several additional physical ingredients that our models include: dust extinction, disk-halo treatment of supernovae-heated gas, and satellite merging. Below we sketch our approach very briefly.

The framework of the semi-analytic approach is the "merging history" of a dark matter halo of a given mass, identified at $z=0$ or any other redshift of interest. We construct Monte-Carlo realizations of the "merger trees" using the method of Somerville \& Kolatt (1998). Each branch in the tree represents a halo merging event. When a halo collapses or merges with a larger halo, we assume that the associated gas is shock heated to the virial temperature of the halo. This gas then radiates energy and cools. The cooling rate depends on the density, metallicity, and temperature of the gas. Cold gas is turned into stars using a simple "recipe", and supernovae energy reheats the cold gas according to another recipe. These two processes are some of the most uncertain elements of these models, and indeed of any attempt to model galaxy formation. As in our investigation of local galaxy properties in SP98, we shall consider several different combinations of recipes for star formation and supernovae feedback.

To model star formation, we use an expression of the general form:

$\dot{m}_{*}=\frac{m_{\text {cold }}}{\tau_{*}}$,

where $\dot{m}_{*}$ is the star formation rate, $m_{\text {cold }}$ is the total mass of cold gas in the disk, and cold gas is converted into stars with a time-scale $\tau_{*}$. In principle this time-scale could be a function of the circular velocity or dynamical time of the disk or other variables. The simplest version of this recipe takes $\tau_{*} \equiv \tau_{*}^{0}=$ constant; i.e. the efficiency of conversion of cold gas into stars $\dot{m}_{*} / m_{\text {cold }}$ is constant over the lifetime of the galaxy. We shall refer to this as the "constant efficiency" recipe (SFR-C).

A recipe that has been used by CAFNZ94 and subsequent papers by this group (henceforth referred to as the "Durham" group) treats the efficiency of conversion of cold gas into stars as a power law function of the circular velocity of the galaxy, with no explicit dependence on redshift:

$\dot{m}_{*}=\frac{m_{\text {cold }}}{\tau_{*}^{0}\left(V_{c} / V_{0}\right)^{\alpha_{*}}}$

Here $V_{c}$ is the circular velocity of the galaxy, $V_{0}$ is a normalization factor, and $\tau_{*}^{0}$ and $\alpha_{*}$ are free parameters. Thus a galaxy with a given circular velocity and cold gas mass will have the same star formation rate at any redshift. 
In KWG93 and in subsequent papers by this group (henceforth referred to as the "Munich" group), the star formation efficiency scales as the inverse of the dynamical time of the galaxy:

$\dot{m}_{*}=\frac{m_{\text {cold }}}{\tau_{*}^{0} \tau_{\text {dyn }}}$,

where $\tau_{*}^{0}$ is a free parameter, and $\tau_{\text {dyn }}=r_{\text {disk }} / V_{c}$ is the dynamical time of the disk. Hierarchical clustering models predict that galaxies are more concentrated at high redshift, therefore galaxies with a given circular velocity are smaller and so the dynamical time is shorter $\left(\tau_{\text {dyn }} \propto(1+z)^{-1.5}\right.$ for $\Omega=1$ ). This means that the star formation efficiency will be higher at high redshift for a galaxy with a given circular velocity and cold gas mass (see SP98, Fig. 1). This star formation rate corresponds to one of the global recipes suggested by Kennicutt (1998) based on observations of local galaxies.

For historical reasons and in order to have brief labels for things, we shall refer to the star formation recipe summarized in Eqn. 2 as the "Durham" recipe (SFR-D) and Eqn. 3 as the "Munich" star formation recipe (SFR-M).

Both the Munich and Durham groups model gas reheating by supernovae feedback using a power law function of the halo circular velocity. The rate of reheating of cold gas is given by

$\dot{m}_{\mathrm{rh}}=\epsilon_{\mathrm{SN}}^{0}\left(V_{c} / V_{0}\right)^{-\alpha_{\mathrm{rh}}} \dot{m}_{*}$

where $\epsilon_{\mathrm{SN}}^{0}$ and $\alpha_{\mathrm{rh}}$ are free parameters, $\dot{m}_{*}$ is the star formation rate, and $V_{0}$ is an arbitrary factor chosen so that $\epsilon_{\mathrm{SN}}^{0}$ is of order unity. The Munich group assumes $\alpha_{\mathrm{rh}}=2$, whereas the Durham group assumes a considerably stronger dependence on circular velocity, $\alpha_{\mathrm{rh}}=5.5$, because they find that this is what is necessary to obtain a local luminosity function with a flat enough faint end slope.

The "reheated" gas is removed from the cold gas reservoir. An important issue is whether the reheated gas then remains in the halo in the form of hot gas, where it will generally cool again on a short time-scale, or is expelled from the potential well of the halo entirely. The two obvious extremes are either to expell all the reheated gas from the halo, or to retain it all it inside the halo. In SP98, we attempted to develop a physically motivated middle ground, which we called the "disk-halo" model. In this model, the amount of gas that is removed from the disk, and the amount that is expelled from the halo are each calculated based on the energy deposited in the gas by the supernovae and the escape velocity of the disk and halo respectively. We showed in SP98 that introducing this disk-halo feedback model improves the agreement with several important local observations, such as the faint end slope of the luminosity function, the metallicity-luminosity relation for dwarf galaxies, and the slope of the small-mass end of the $\mathrm{H}_{\mathrm{I}}$ mass function.

As in SP98, we will investigate different combinations of star formation and feedback recipes to illustrate how these affect our results. We refer to these combinations, summarized in Table 1, as the Durham, Munich, and Santa Cruz "packages". Although we use the same recipes for quiescent star formation and supernovae feedback, we emphasize that many other aspects of our models differ from the published models of the Munich and Durham groups, and we are not trying o reproduce their results in detail. Our goal is to illustrate the differences arising from different ways of treating the star formation and supernovae feedback in the models. The predicted properties of galaxies at $z=0$ in these different packages are discussed in detail in SP98. The one exception is the "Santa Cruz 1" package, which is not shown in SP98. The only difference between this package and the fiducial Santa Cruz package presented in SP98 (which we refer to here as "Santa Cruz 2") is the recipe used for quiescent star formation. We find that the properties of local $(z=0)$ galaxies (such as the luminosity function and Tully-Fisher relation) for both Santa Cruz packages are almost identical.

Each new generation of stars produces a fixed yield of metals, which are recycled through the cold and hot gas phase as the gas cools and is reheated by supernovae. The feedback model also affects the chemical evolution, depending on what fraction of the metals produced are ejected from the halo or retained in the hot gas. In our Munich and Santa Cruz packages, newly produced metals suffer the same fate as the reheated gas (i.e., are mixed with the hot gas or ejected from the halo) and in the same proportions. In our Durham package, the new metals are initially mixed with the cold gas; they can then be ejected from the halo along with the next batch of reheated gas.

When dark matter halos merge, the galaxies contained within them survive and may merge on a longer time-scale. After a halo merger event, the central galaxy of the largest progenitor halo becomes the new central galaxy, and all other galaxies become "satellites". Satellite galaxies may merge with the central galaxy on a dynamical friction timescale, or with each other on approximately a mean free path time-scale. However, if the relative velocities of the satellites are large compared to their internal velocities, they will not experience a binding merger. The modelling of galaxy merging is discussed in more detail in Section 2.2 Following a merger event between two galaxies with similar masses $\left(m_{\text {smaller }} / m_{\text {larger }}>f_{\text {bulge }}\right)$, the stars and gas of both are placed in a "bulge" component. $f_{\text {bulge }}$ is adjusted in order to obtain the correct relative fraction of elliptical, S0, spiral and irregular galaxies (see SP98); typically, $f_{\text {bulge }} \sim 0.3$. Subsequent cooling and star formation may then form a new disk. Thus galaxies may be assigned morphologies based on their bulge-to-disk ratio. The star formation history of each galaxy is convolved with stellar population models (e.g., Bruzual \& Charlot 1993) to obtain magnitudes in any desired filter band. A fraction $f_{\text {lum }}$ of the stellar mass is assumed to be in luminous stars, with the remainder assumed to be in the form of non-luminous baryonic dark matter (brown dwarfs). In our models, we typically require $f_{\text {lum }}=0.9$ to 1.0 in order to satisfy our normalization requirements (explained in the following paragraph).

As explained in detail in SP98, the four key free parameters - those describing the star formation rate $\left(\tau_{*}^{0}\right)$, the supernovae feedback efficiency $\left(\epsilon_{\mathrm{SN}}^{0}\right)$, the metallicity yield $(y)$, and the luminous fraction of the stars $\left(f_{\text {lum }}\right)$ - are set by requiring an average fiducial "reference galaxy" (the central galaxy in a halo with a circular velocity of $220 \mathrm{~km} \mathrm{~s}^{-1}$ ) to have (a) an I-band magnitude $M_{I}-5 \log h=-21.7$, (b) a cold gas mass $m_{\text {cold }}=1.25 \times 10^{10} h^{-2} M_{\odot}$, and (c) a stellar metallicity of about solar. The first requirement fixes the zero-point of the I-band Tully-Fisher relation so that it agrees with observations Giovanelli et al. 1997; Willick et al. 1995; Willick et al. 1996). Requirement (b) fixes the 
Table 1. Star Formation/Feedback Packages

\begin{tabular}{lccc}
\hline name & quiescent star formation & feedback & reheated gas \\
\hline Durham & SFR-D (Eqn. 2 & Eqn. A $\alpha_{\text {rh }}=5.5$ & all ejected from halo \\
Munich & SFR-M (Eqn. 3) & Eqn.,$\alpha_{\text {rh }}=2$ & all stays in halo \\
Santa Cruz 1 & SFR-C (Eqn. 1, $\tau_{*}=$ constant) & disk/halo model & according to disk/halo model \\
Santa Cruz 2 & SFR-M (Eqn. 3) & disk/halo model & according to disk/halo model \\
\hline
\end{tabular}

cold gas content of the "reference galaxy" to agree with observed $\mathrm{H}_{\mathrm{I}}$ masses, allowing for a contribution from molecular hydrogen. These three requirements allow some freedom in choosing the four key parameters; this freedom may be exploited by adjusting the supernovae feedback efficiency to obtain the best agreement with both the observed TullyFisher relation and the observed local luminosity function (see SP98).

Note that the free parameters have been set entirely by comparing to present-epoch observations. We find that reasonably good agreement with a broad range of $z \sim 0$ observations may be obtained with any of several different recipes for star formation and supernovae feedback (see SP98). Now we add a time dimension by investigating the predictions of the models for high-redshift observations. As we shall see, this comprises a much stronger constraint on the models, and may provide indications of areas in which new physics should be included, or where the current treatment of certain processes should be modified.

\subsection{Modelling Starbursts}

Previous work using semi-analytic models has not systematically investigated the importance of a bursting mode of star formation, particularly its effect on the interpretation of the observations of high-redshift galaxies. We start with the ansatz that galaxy-galaxy mergers trigger starbursts. This premise has considerable observational support, and is also supported by N-body simulations with gas dynamics. Mihos \& Hernquist 1994, 1996 have simulated galaxy-galaxy mergers using a high resolution N-body/smoothed particle hydrodynamics code (TREESPH) with star formation modelled according to a Schmidt law $\left(\rho_{S F R} \propto \rho_{\text {gas }}^{n}\right.$ with $\left.n=1.5\right)$. In Mihos \& Hernquist (1996), mergers between equal mass galaxies (major mergers) were simulated. When the galaxies have no bulge component, they found that the burst starts soon after the initial encounter, while the galaxies are still physically separated in space. The star formation rate in each galaxy reaches a peak of 20-30 times its pre-merger amplitude, and an order of magnitude increase is sustained for $\sim 150$ Myr. In mergers where one galaxy has a bulge component with one-third the mass of the disk, they find that the peak of the starburst is delayed until the galaxies actually merge, but the ensuing starburst is more intense, reaching a peak of more than 70 times the pre-merger rate in each galaxy. This rapidly depletes the gas supply, resulting in a shorter burst time-scale of about $50 \mathrm{Myr}$. However, Mihos \& Hernquist (1996) emphasize that, although the details of the starburst behaviour are rather different depending on the morphology of the galaxy, the time-integrated result is nearly identical. In both cases (with and without a bulge), about 75 percent of the total gas supply is converted into stars over the course of the burst. Some of the remaining gas is launched to large distances in the form of tidal tails, and some (a few times $10^{9} M_{\odot}$ ) remains in the disk. This result remains relatively consistent over various permutations of the encounter geometry (i.e., prograde-prograde, prograderetrograde, retrograde-retrograde, and tilted orbits), with the fraction of gas consumed in the burst varying from 65 to 85 percent.

The case of highly unequal mass mergers was explored in Mihos \& Hernquist (1994). The case they simulated represents a "Milky Way" sized disk galaxy accreting a satellite that is one tenth of its mass. The nonaxisymmetric mode generated by the accretion of the satellite causes a large fraction of the gas to collapse into the central region of the galaxy, fueling a strong starburst. In this case about 50 percent of the original gas supply is consumed in the starburst, which lasts for about $60 \mathrm{Myr}$. However, the results are much more sensitive to the morphological structure of the galaxies. If the larger galaxy has a bulge (the case simulated has a bulge to disk ratio of $1: 3$ ), the bulge seems to stabilize the disk against strong radial gas flows, leading to a much weaker starburst event (only about 5 percent of the total gas supply is consumed). Mihos \& Hernquist (1994) note that this implies that the burst rate will decrease at low redshift, as galaxies develop bulges, even if the merger rate remains constant.

We now introduce a simple model for starbursts based on the results described above. In our models, galaxies that are within the same halo may merge according to two different processes:

- Central merger. Satellite galaxies lose energy and spiral in to the center of the halo on a time-scale given by the dynamical friction time-scale (Binney \& Tremaine 1987):

$\tau_{\mathrm{df}}=\frac{1.17}{\ln \Lambda} \frac{r_{\mathrm{vir}}^{2} V_{c}}{G m_{\mathrm{sat}}}$

Here $r_{\text {vir }}$ is the virial radius, $V_{c}$ is the circular velocity, and $\ln \Lambda$ is the usual Coulomb logarithm, which we approximate as $\ln \Lambda \approx \ln \left(1+m_{h}^{2} / m_{\text {sat }}^{2}\right)$, where $m_{h}$ is the mass of the parent halo and $m_{\text {sat }}$ is the mass of the sub-halo containing the satellite galaxy. Each satellite merges with the central galaxy after a time $\tau_{\mathrm{df}}$ has elapsed.

- Satellite merger. In addition, satellite galaxies orbiting within the same halo may merge with one another according to a modified mean free path time-scale:

$$
\begin{array}{r}
\tau_{\mathrm{mfp}}=1.25\left(\frac{N}{20}\right)^{-2}\left(\frac{r_{\text {halo }}}{\mathrm{Mpc}}\right)^{3}\left(\frac{r_{\text {gal }}}{0.12 \mathrm{Mpc}}\right)^{-2} \\
\left(\frac{\sigma_{\text {gal }}}{100 \mathrm{~km} \mathrm{~s}^{-1}}\right)^{-4}\left(\frac{\sigma_{\text {halo }}}{300 \mathrm{~km} \mathrm{~s}^{-1}}\right)^{3} \mathrm{Gyr} .
\end{array}
$$


Here $r_{\text {halo }}$ is the virial radius of the parent halo, $r_{\text {gal }}$ is the tidal radius of the sub-halo containing the satellite galaxy, $\sigma_{\text {gal }}$ is the internal 1-D velocity dispersion of the galaxy, and $\sigma_{\text {halo }}$ is the 1-D velocity dispersion of the parent halo. This expression is the scaling formula derived by Makino \& Hut (1997) to describe the results of dissipationless N-body simulations in which galaxy-galaxy encounters and mergers covering a large region of parameter space were simulated. Note that in this expression, the merger rate $\tau_{\mathrm{mfp}}^{-1}$ increases with the square of the number of galaxies in the halo and with the halo density, as expected, and decreases as the cube of the ratio of the galaxy's internal velocity to the velocity dispersion of the halo. This reflects the fact that if the relative velocity of the galaxies is much larger than their internal velocities, they will not experience a binding merger. Thus, maximum satellite-satellite merger rates occur in halos with circular velocities of about $500 \mathrm{~km} \mathrm{~s}^{-1}$, or about the size of a group.

When any two galaxies merge, the "burst" mode of star formation is turned on. We then model the star formation rate during the burst as a Gaussian function of time. The burst model has two adjustable parameters, the time-scale of the burst $\left(\sigma_{\text {burst }}\right)$ and the efficiency of the burst $\epsilon_{\text {burst }}$. The efficiency $\epsilon_{\text {burst }}$ is defined as the fraction of the cold gas reservoir (of both galaxies combined) that is turned into stars over the entire duration of the burst.

As in SP98, we classify the merger as "major" or "minor", according to whether the ratio of the smaller to the larger of the galaxies' baryonic masses is greater than or less than the value of the parameter $f_{\text {bulge }} \sim 0.3$. In this paper, we use $\epsilon_{\text {burst }}=0.7$ and $\sigma_{\text {burst }}=32.5$ Myr for major mergers, and we use $\epsilon_{\text {burst }}=0.5$ and $\sigma_{\text {burst }}=50$ Myr for minor mergers. In major mergers, all the existing stars of both galaxies, along with all new stars formed in the burst, are placed in a bulge component. In minor mergers, the pre-existing stars from the smaller galaxy are placed in the disk component of the post-merger galaxy, but the stars formed in the burst are placed in the bulge. The former is motivated by the N-body simulations of similar satellite accretion events by Walker. Mihos, \& Hernquist (1996), which show that 90 percent of the satellite mass is stripped and distributed in the disk of the larger galaxy, and only the densest part of the satellite core ends up at the center. If the bulge-to-disk ratio of the larger galaxy exceeds 0.3 , we decrease the burst efficiency to $\epsilon_{\text {burst }}=0.05$. We have chosen the values of the burst parameters in order to roughly reproduce the behaviour observed in the simulations of Mihos \& Hernquist (1994) and Mihos \& Hernquist (1996), described above.

A possible problem in scaling from these $z=0$ simulations is that at $z \sim 3$ many of the important properties of galactic disks are probably quite different. The galaxies are smaller and denser, and the burst time-scales could be shorter. The disks may be less stable because they have a higher gas fraction, or more stable because the disk to dark matter halo mass ratio may be smaller. If the burst scenario proves to be interesting, a more detailed treatment of starbursts at high redshift should be developed using a more extensive set of N-body hydrodynamic simulations similar to those of Mihos \& Hernquist. Similarly, our model for satellite-satellite mergers $\left(\tau_{\mathrm{mfp}}\right)$ and infall and tidal stripping of sub-halos should be refined and tested against high-

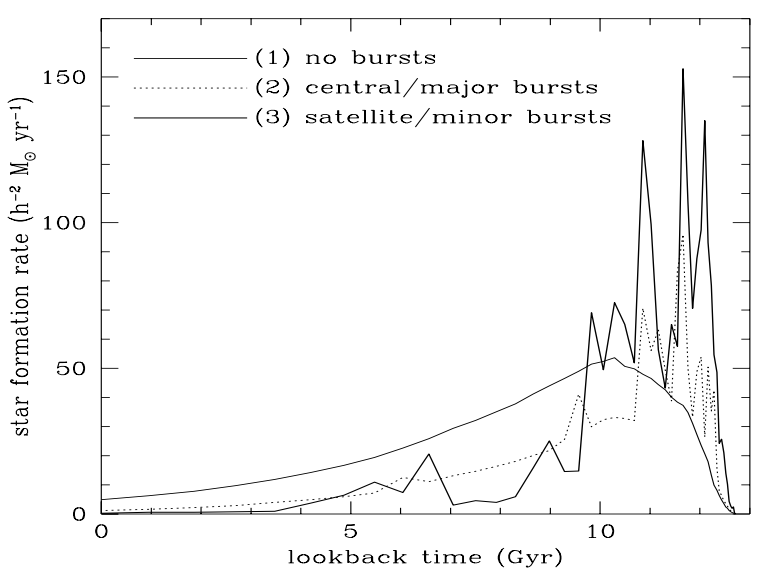

Figure 1. The total star formation rate for all galaxies that end up within a halo with $V_{c}=500 \mathrm{~km} \mathrm{~s}^{-1}$ at $z=0$ for SCDM, using the constant star formation recipe SFR-C. The thin solid line shows a model (1) with no starbursts, the dotted line shows a model (2) with mergers only between satellite and central galaxies and bursts only in major mergers, and the bold solid line shows a model (3) with satellite-satellite and satellite-central mergers and bursts in major and minor mergers. The lookback time is computed for an $\Omega=1$ model with $H_{0}=50 \mathrm{~km} \mathrm{~s}^{-1} \mathrm{Mpc}^{-1}$. Note that in model 3 the peaks, representing starbursts, occur primarily at lookback times of 8 to 12 Gyr (redshifts $z \sim 2-5$ ).

resolution dissipationless cosmological simulations. Work on both of these issues is in progress.

Fig. 11 shows the total star formation rate for all the galaxies in a "group" sized halo $\left(V_{c}=500 \mathrm{~km} \mathrm{~s}^{-1}\right.$ at $\left.z=0\right)$. We show the star formation rate in a model with: (1) no starbursts (quiescent star formation only), (2) bursts in major mergers only and satellite galaxies allowed to merge only with the central galaxy on a dynamical friction time-scale (satellite-central mergers), and (3) both satellite-central and satellite-satellite mergers, and bursts in both major and minor mergers. The models of BCFL98 contain starbursts in a similar way to model 2 (see the last two sentences in the fourth paragraph of their $\S 6$ ), except that in their models it is assumed that the bursts are 100 percent efficient and are instantaneous (Baugh, Cole, \& Frenk 1996), rather than having a gas consumption efficiency $\epsilon_{\text {burst }}<1$ and a finite time-scale $\sigma_{\text {burst }}$ as in our models. Fig. 1 shows that the star formation rate at high redshift is considerably amplified in model 3 compared to model 2 , illustrating that neglecting satellite-satellite mergers and bursts in minor mergers will considerably underestimate the importance of starbursts at high redshift. The burst models discussed in the remainder of this paper correspond to model (3) above.

The free parameters are re-tuned for each set of model ingredients, i.e. the star formation and supernovae feedback recipe, cosmology, and inclusion of starbursts, according to the constraints discussed in Section 2.1 and SP98. In practice, the values of the parameters are not dramatically different in the models with and without starbursts. Once the parameters have been adjusted in this way, the mean properties of galaxies at $z=0$ (such as the luminosity function and Tully-Fisher relation) are not significantly affected by the inclusion of starbursts. The most noticeable effect of the inclusion of starbursts on the predicted properties of local 
galaxies is an increase in the scatter in the Tully-Fisher relation, colour-magnitude relation and other relations.

\section{MODEL RESULTS}

In this section, we are mainly interested in showing how the inclusion of starbursts affects the properties of galaxies at high redshift. Throughout this section, the models are set within the SCDM cosmology and use the "Santa Cruz 1" package for quiescent (non-burst) star formation and supernovae feedback. In this package, we make the simple assumption that quiescent star formation has a constant efficiency $\left(\tau_{*}=\right.$ constant in Eqn. 1; SFR-C).

We have used SFR-C in this section both because it represents the simplest possible "null hypothesis" about star formation, and because it leads to the best overall agreement with the observations including the evolution of global quantities such as the cold gas and metal content of the Universe. In the final section of the paper, we shall argue that this simple recipe may give similar results to a more physically motivated and detailed model of quiescent star formation. Throughout this section, we use a standard Salpeter IMF (Salpeter 1955) and the solar metallicity model SEDs from the GISSEL95 models of Bruzual \& Charlot (1993). In the following section, we show the results of changing some of these ingredients.

Because extensive spectroscopic follow-up work has now verified the effectiveness of the Lyman-break or "drop-out" technique for finding $z \gtrsim 2$ galaxies, we do not attempt to mimic the color selection process used to identify real Lyman-break objects, but rather assume that all galaxies brighter than a limiting magnitude in our models correspond to "LBGs". The modelling work of BCFL98 has shown that using the same colour selection criteria as the observations picks out model galaxies in the expected redshift range.

We do not include the effect of absorption by intervening cold gas clouds, because although this effect is very important shortwards of the Lyman limit, for redshifts less than $z \sim 4$ it does not much affect the spectral energy distribution (SED) in the wavelength range relevant to our study. We have used the filter response functions of the WFPC2 filters F300W and F814W ( $V_{606}$ and $I_{814}$, with effective wavelengths of $\sim 6000$ and $\sim 8000 \AA$ respectively) with zeropoints corresponding to the $\mathrm{AB}$ magnitude system. Where necessary, conversions from $\mathrm{ST}$ to $\mathrm{AB}$ magnitudes have been performed using the information provided in the HDF website (http://www.stsci.edu/ftp/science/hdf/logs/zeropoints.txt). We approximate the $\mathcal{R}$ filter (Steidel \& Hamilton 1992) used in the ground based observations by $\left(V_{606}+I_{814}\right) / 2$ (Dickinson 1998). At $z \sim 3$, this corresponds to a rest wavelength of about $1600 \AA$.

\subsection{Comoving Number Density}

We first investigate whether the models reproduce the observed number densities of objects. Fig. 2 shows the comoving number density of galaxies brighter than a fixed magnitude limit as a function of redshift, over the redshift range probed by the observed LBGs. We show this function using three values for the magnitude limit: the top panel shows

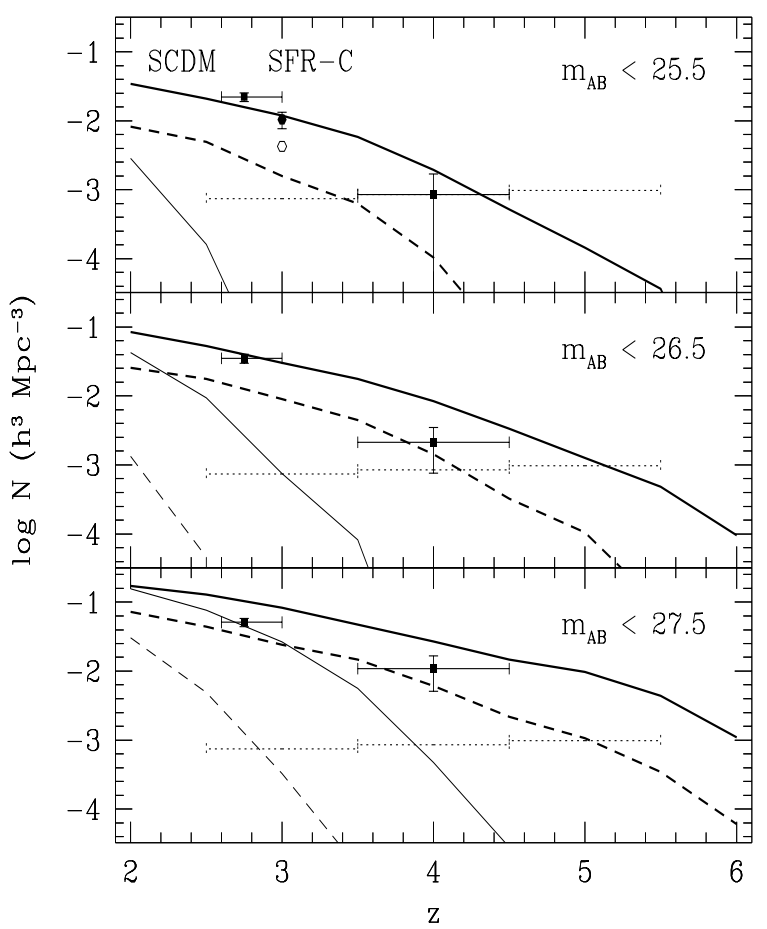

Figure 2. The comoving number density of galaxies brighter than $m_{\mathrm{AB}}=25.5$ (top panel), 26.5 (middle panel), or 27.5 (bottom panel $)$, where $m_{\mathrm{AB}}=\left(V_{606}+I_{814}\right) / 2$. The open hexagon indicates the comoving number density of LBGs with spectroscopic redshifts from the ground-based sample (Giavalisco et al. 1998). The filled hexagon at $z=3$ indicates the number density of $\mathrm{U}$ dropouts in the HDF with spectroscopic redshifts larger than 2.5 (and brighter than 25.5) (Dickinson 1998). The filled squares at $z=2.75$ and $z=4$ indicate the number density of $\mathrm{U}$ and $\mathrm{B}$ drop-outs (respectively) in the HDF (Pozzetti et al. 1998). See text for details on how the number densities were calculated. The dashed horizontal lines correspond to one galaxy in the HDF volume for the indicated redshift intervals. Bold solid lines show the comoving number density of galaxies in the SCDM models with starbursts; light solid lines show the results of the no-burst models. Dashed lines show the result of reducing the flux of each galaxy by a factor of three to estimate the effects of dust extinction. No-burst models underproduce LBGs, especially at bright magnitudes and high redshift. The disagreement is dramatic if there is really a factor of three extinction due to dust.

the abundance of bright galaxies $\left(m_{\mathrm{AB}}<25.5\right)$, the middle panel shows the abundance of galaxies brighter than 26.5, and the bottom panel shows galaxies brighter than 27.5. Here we have used $m_{\mathrm{AB}}=\left(V_{606}+I_{814}\right) / 2$ for the models and for counting the HDF galaxies. This is comparable to the $\mathcal{R}$ filter used for the ground-based observations (Dickinson 1998). We have calculated the comoving number density for the ground-based sample of LBGs with spectroscopic redshifts using the average of the surface densities for the nine fields presented in Table 1 of Giavalisco et al. (1998), $\mathcal{N}=1.21 \pm 0.15 \operatorname{arcmin}^{-2}$. The $1 \sigma$ field-to-field variance is about 12 percent. If plotted on Fig. 2, the error bar would be smaller than the symbol. The quoted magnitude limit for these observations is $\mathcal{R}<25.5$. We parameterize the selection function as a Gaussian function of redshift, with a mean 
redshift $\bar{z}=3$ and $\sigma_{z}=0.27$. This is a good approximation to the selection function shown in Fig. 1 of Giavalisco et al. (1998). In deriving the number density, we have assumed 100 percent efficiency at the peak of the selection function. This is likely to underestimate the actual number density as some galaxies were almost certainly missed from the spectroscopic sample. It should be noted that BFCL98 compared their predictions to the projected number density given by Steidel et al. (1996b), $\mathcal{N}=0.4 \pm 0.07 \operatorname{arcmin}^{-2}$ to $\mathcal{R}=25$. Although this was the best data available at the time, the results from the much more extensive data now available are about a factor of three larger though the sample goes only about half a magnitude fainter.

We have calculated the comoving number density of galaxies at $z \sim 3$ and $z \sim 4$ in the HDF using the list of $\mathrm{U}$ and B drop-outs from Table 1 of Pozzetti et al. (1998). The procedure for identifying drop-outs is similar to that used by Madau et al. (1996), although Pozzetti et al. (1998 have made more stringent requirements about signal-to-noise in identifying the objects. The selection function for the HDF LBGs is not very well understood because spectroscopic redshifts have been obtained for only 26 objects at $z>2$ (Dickinson 1998). The filters differ from those used for the groundbased work, and probably the selection function is somewhat different. In particular, the mean redshift of the HDF sample is thought to be somewhat lower than that of the groundbased sample ( $\bar{z}=2.75$ according to Madau et al. (1996), $\bar{z}=2.7$ for the galaxies with measured redshifts, and according to Dickinson (1998) $\bar{z}$ may perhaps be as low as 2.6 for the complete photometric sample). Although, from Fig. 7 of Dickinson (1998), which shows the redshift distribution of galaxies with spectroscopic redshifts in the HDF and Flanking Fields, if the large "spike" at $z \sim 2.4$ were removed, the selection function looks like it would be quite similar to that of the ground based sample.

For the HDF, we have therefore calculated the comoving number density in several ways, and find that compared to the large uncertainties in the modelling, all of the estimates are relatively similar. For example, we first convert the $V_{606}(\mathrm{ST})$ magnitudes of Pozzetti et al. (1998) to our $\left(V_{606}+\right.$ $\left.I_{814}\right) / 2(\mathrm{AB})$ system using the mean $V_{606}-I_{814}$ colours from the lists of $\mathrm{U}$ and $\mathrm{B}$ dropouts in Madau et al. (1996) and the $(\mathrm{ST}-\mathrm{AB})$ conversion from the HDF website. We then find $29.9 \mathrm{U}$ drop-outs brighter than $\left(V_{606}+I_{814}\right) / 2=25.5$ (AB). This gives a surface density of $\mathcal{N}=6.36 \mathrm{arcmin}^{-2}$ (n.b. about a factor of five higher than the ground-based spectroscopic sample). If we use the same selection function as the ground-based sample, we obtain a comoving number density of $n=2.25 \times 10^{-2} h^{-3} \mathrm{Mpc}^{3}$. If we assume that the galaxies are uniformly distributed in the redshift interval $2.5<z<3.5$, we obtain $n=2.23 \times 10^{-2} h^{-3} \mathrm{Mpc}^{3}$. Assuming a lower bound for the redshift interval of $z=2.2$ or $z=$ 2.0 leads to number densities of $n=1.68 \times 10^{-2} h^{-3} \mathrm{Mpc}^{3}$ and $n=1.44 \times 10^{-2} h^{-3} \mathrm{Mpc}^{3}$, respectively. The difference between the smallest and largest of these estimates is about 0.2 in the $\log$, or one small tickmark on our figure - quite unimportant compared to the current uncertainties in the modelling. We have therefore assumed that the galaxies are uniformly distributed in the redshift interval $2.5 \leq z \leq 3.5$; i.e. we have simply divided the number of drop-outs by the comoving volume of the HDF in this interval. We have placed the symbol at the mean redshift $\bar{z}=2.75$ of the full photometric sample according to Madau et al. (1996), and indicated what we believe to be the uncertainty in the mean redshift with the horizontal error bar. We have also shown the number density of bright HDF galaxies with spectroscopically confirmed redshifts $z \geq 2.5$ from Dickinson (1998), for which the mean redshift is $\bar{z} \sim 3$. The comoving number density at $\bar{z}=4.0$ is computed in a similar way, assuming the galaxies are uniformly distributed in the interval $3.5 \leq z \leq 4.5$. The dashed horizontal lines indicate the inverse comoving volume $V_{\text {comoving }}^{-1}$ in the redshift interval indicated; this corresponds to one galaxy in an HDF volume. The redshift intervals spanned by these lines correspond roughly to the redshift intervals where one expects to find $\mathrm{U}, \mathrm{B}$, and $\mathrm{V}$ drop-outs.

Fig. 2 shows that the models with no burst mode seriously underpredict the number density of galaxies, especially at the brightest magnitude limit $m=25.5$. When starbursts are included, the models reproduce or exceed the observed number densities of LBGs when dust extinction is neglected. The inclusion of starbursts causes a bigger change in the comoving number density of LBGs at higher redshifts and at brighter magnitude limits, and so the number densities in the burst models tend to have flatter dependences on both redshift and magnitude. By a redshift of $\sim 2$, the starbursts have a minor effect on the number counts, and by $z=0$ starbursts are unimportant compared to the quiescent star formation mode. This is because the galaxy-galaxy merger rate is larger at high redshifts because the halos are denser, and the starbursts are more dramatic because the galaxies are relatively gas rich.

So far we have not discussed the effects of dust extinction. However, the observed colours of the LBGs, as well as comparison of the $\mathrm{UV}$ to $\mathrm{H} \beta$ fluxes, indicate that there is almost certainly some dust in these galaxies Pettini et al. 1997b; Dickinson 1998). However, the amount of dust and the resulting extinction is quite uncertain. These depend on the metallicity and age of the galaxy, the geometry and "clumpiness" of the dust, and the wavelength dependence of the attenuation law. These issues are discussed with reference to the LBGs by Calzetti (1997a), Pettini et al. (1997b), and Dickinson (1998). The correction factors suggested by these authors typically range from a factor of $\sim 2$ to $\sim 7$, with a mean value of about a factor of three. More dramatic corrections, as large as a factor of $\sim 15$, have been suggested by Meurer et al. (1997), based on extinction relations calibrated for local starbursts, and by Sawicki \& Yee (1998), based on optical and infrared photometry compared to model SEDs.

We have made a simplified estimate of the effect of dust in Fig. 2 by simply decreasing the luminosity of each galaxy by a factor of three. However, according to any physical dust model, a uniform correction by a fixed factor is probably unrealistic. If dust traces metal production (hence star formation activity), more intrinsically luminous galaxies will be more heavily extinguished. There is observational evidence that this is in fact the case for $z \sim 0$ galaxies Giovanelli et al. 1995, Tully et al. 1998). This will lead to an even larger deficit of bright galaxies in the no-burst models seen in Fig. 2. However, if most of the bright galaxies are starbursting objects, as in the burst models, the situation is less clear. The observational work by Calzetti (1997b) and Calzetti, Kinney, \& Storchi-Bergman (1994) indicates that 


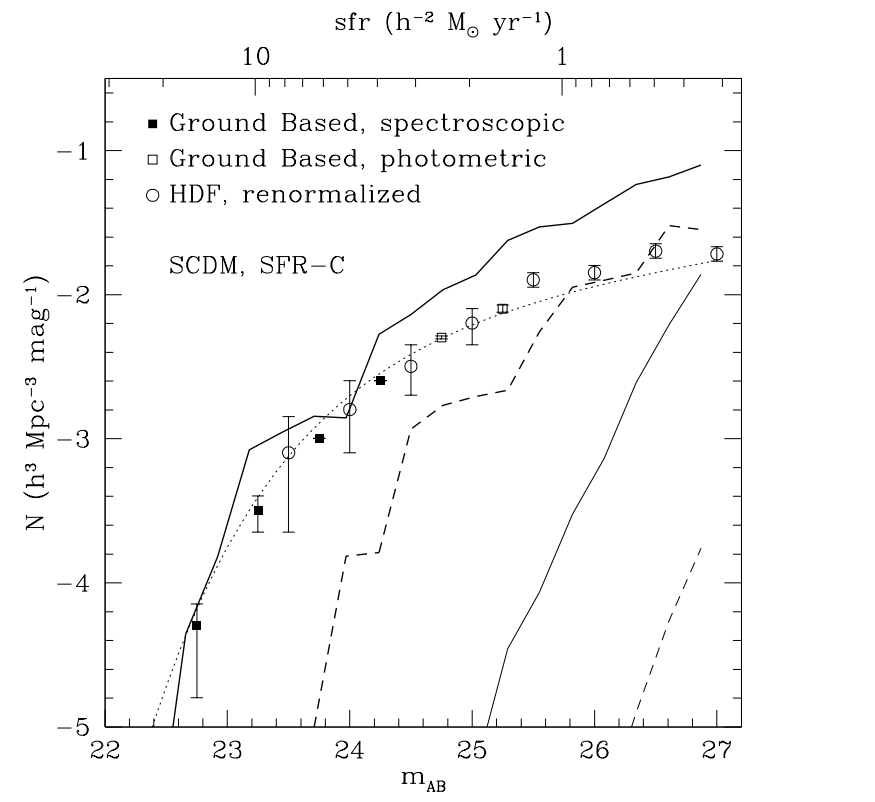

Figure 3. The luminosity function at $z=3$ in the SCDM cosmology. Svmbols show the composite luminosity function derived by Dickinson (1998, see text) from the combined ground-based and HDF samples, and the dotted line shows the Schechter fit to these results. Filled squares are from the ground-based data with spectroscopic redshifts, open squares are from the groundbased data with photometric redshifts, and open circles are from the HDF (photometric) data, renormalized to match up with the ground-based data (see text). Bold lines indicate the results of the models with starbursts, and light lines indicate the results of the no-burst models. The dashed lines show the results of a factor of three correction for dust extinction. The conversion from $L_{1500}$ to star formation rate (shown on the top axis) is discussed in Section 3.3 of the text.

the wavelength dependence of the attenuation due to dust is "greyer" (less steep) in the UV for local starburst galaxies than a Galactic or SMC-type extinction curve. Powerful starbursts could blow holes in the dust, or especially in small objects, perhaps eject much of the metals and dust from the galaxy altogether. On the other hand, regions of active star formation may be completely enshrouded in dust, leading to even stronger extinction. In any case, models without starbursts appear to have no hope of reproducing the observed abundance of bright galaxies at high redshift with even the relatively conservative factor of three correction for dust extinction.

\subsection{Luminosity Function}

In addition to the dependence of the galaxy number density on redshift, it is also important to consider the dependence on galaxy luminosity at a fixed redshift. The bright end $\left(m_{A B} \gtrsim 25.5\right)$ of the luminosity function at $\sim 1500 \AA$ for the observed LBGs at $z \sim 3$ can be determined fairly accurately from the relatively large ground-based spectroscopic sample. The HDF can be used to give some indication of the faint end slope, although we should note that there is probably some incompleteness faintwards of about $m=26$. The composite luminosity function constructed in this way (shown in Fig. 9 of Dickinson 1998) is fit remarkably well by a Schechter function with a faint end slope similar to the value derived by Gallego et al. (1995) for local galaxies (it should be noted, however, that the faint end slope of the local UV (2000 A) luminosity function derived by Treyer et al. (1997) is considerably steeper: $\alpha=-1.8$ as compared to $\alpha=-1.3$ from the $\mathrm{H} \alpha$ data of Gallego et al. (1995)). As we mentioned in the previous section, the normalization of the HDF luminosity function is systematically higher than the ground based data, and Dickinson (1998) has renormalized the HDF part of the luminosity function to match up with the ground based data. Thus the amplitude of the luminosity function may be higher than what we have shown in Fig. 3 . The parameters of the Schechter function shown in Fig. 3 are $m_{*}=24.38, \alpha=1.3$ and $\phi_{*}=9.92 \times 10^{-3} h^{3} \mathrm{Mpc}^{-3}$. These are in reasonably good agreement with the parameters derived by Pozzetti et al. (1998), if converted from the $q_{0}=0.05$ cosmology used by these authors to $q_{0}=0.5$.

As already suggested by the comparison of the comoving number density at different magnitude limits, the luminosity function in the no-burst models is not only too low in amplitude, it is too steep; i.e., there are too many faint galaxies compared to the number of bright galaxies. Without dust extinction, the burst models produce a luminosity function that is in fairly good agreement with the observed one, although the faint end slope is a bit steeper. However, with the moderate factor of three dust correction as above, even the burst models produce too few bright galaxies. As we discussed before, a more realistic dust correction would mainly affect the bright galaxies and would probably further steepen the luminosity function.

It should be noted at this point that there is nothing surprising about our finding that in the no-burst models with a constant star formation efficiency, high-redshift galaxies are on average much less luminous than present day galaxies - theories of hierarchical structure formation generically predict that galaxies are considerably smaller and less massive at high redshift. The surprising thing is that the observed LBGs are brighter and more numerous than $z=0$ galaxies (see Fig. 9 of Dickinson 1998; also see Pettini et al. 1997b). Their intrinsic luminosities must be even more impressive if they are affected by significant dust extinction.

\subsection{Star Formation Rates}

The observed rest-frame UV fluxes can be used to derive star formation rates using spectral synthesis models. In Fig. 3, the top axis shows the equivalent star formation rate derived from the UV luminosity (1500 $\AA$ ) at $z=3$ assuming the conversion factor appropriate to a Salpeter $\operatorname{IMF}\left(S F R=L_{\mathrm{UV}} / 8.0 \times 10^{27} \mathrm{ergs} \mathrm{s}^{-1} \mathrm{~Hz}^{-1}\right.$, from Madau, Pozzetti, \& Dickinson 1997). We find that in the no-burst models, the UV luminosities and the actual instantaneous star formation rates in the models lie exactly on the Madau relation with very little scatter (see Fig. (1)), as expected. In the burst models, there is some scatter around this relation, but the UV flux is still a good indicator of the instantaneous star formation rate for the majority of the galaxies. The main relevance of this point is that the UV continuum flux remains elevated for considerably longer after a burst of star formation than the flux from emission lines such as $\mathrm{H} \beta$ and $\mathrm{O}[\mathrm{III}]$. If a significant number of galaxies were bright 


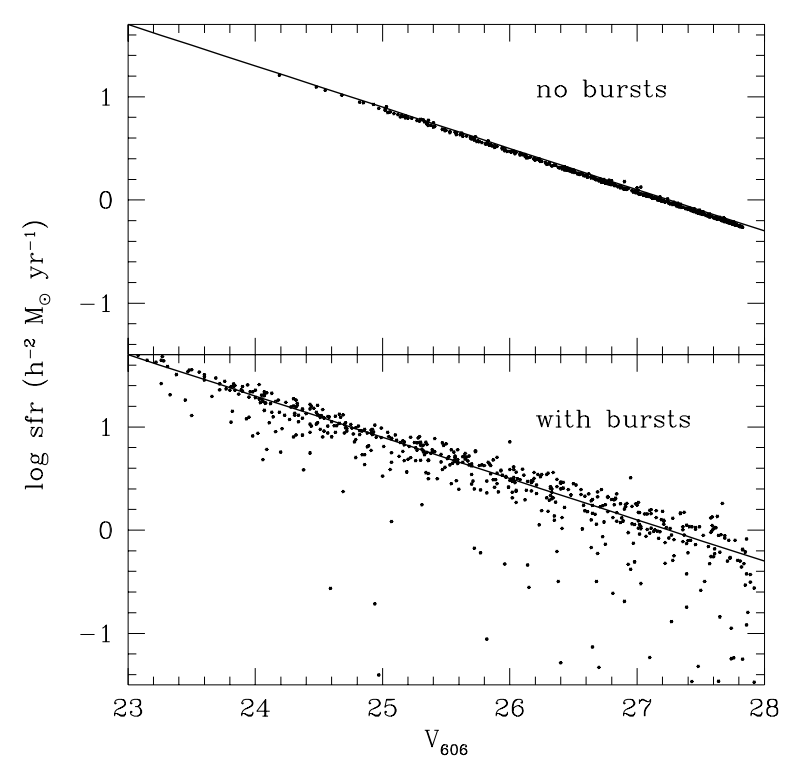

Figure 4. The relationship between UV flux $\left(V_{606, \mathrm{AB}}\right)$ and the instantaneous star formation rate in the no-burst (top) and burst (bottom models. The solid line shows the relation given by Madau, Pozzetti, \& Dickinson (1997). The bottom panel shows that the UV continuum flux remains a good indicator of the instantaneous star formation rate for the majority of galaxies even in the burst models. Thus the burst models are still consistent with the observation that all of the $z \sim 3$ LBGs that have so far been observed in the near-IR have detectable emission lines $(\mathrm{H} \beta$ and $\mathrm{O}[\mathrm{III}])$.

enough in the UV to be detected as LBGs but had low instantaneous star formation rates, one would not expect to observe emission lines in these galaxies. There is an ongoing effort to observe LBGs in the near-IR, where these lines are expected to be visible for $z \sim 3$ objects. So far, nearIR observations have been attempted for only a few of the brightest objects, but every object that has been observed shows detectable emission lines (Pettini et al. 1998). From Fig. 4, we see that this is not inconsistent with the predictions of the burst models.

\subsection{Stellar Masses, Radii, and Line-widths}

Fig. 5 shows the stellar masses of objects with $\mathcal{R}<25.5$ at $z=3$. In the no-burst models, only objects with masses of about $10^{10} h^{-1} M_{\odot}$ are bright enough to attain this luminosity. In the burst models, a significant number of smaller objects (as small as $\sim 10^{9} h^{-1} M_{\odot}$ ) become bright enough to be included in the sample. In Fig. 6 we show the effective radius (baryonic half mass radius) of the model galaxies as a function of their circular velocity (see Section 2.3 of SP98 for a discussion of how sizes are estimated in our models). The circular velocity is the virial circular velocity of the last halo in which the galaxy was a central galaxy. The sizes of the bright galaxies in both the burst and no-burst models are in reasonable agreement with the average half-light radii of the LBGs observed in the HDF (Giavalisco, Steidel, \& Macchetto 1996; Lowenthal et al. 1997). The sizes of the model galaxies at $z=0$ are also in good agreement with the sizes of

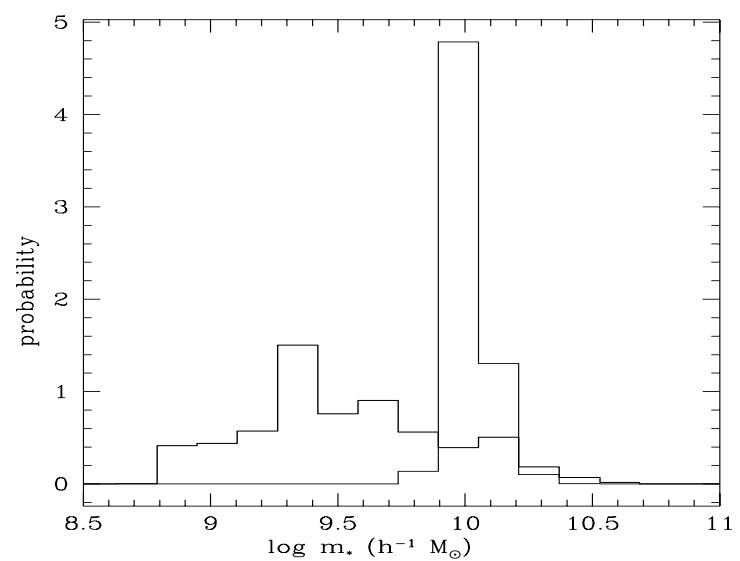

Figure 5. The probability distribution for the stellar masses of the model "LBGs" $(\mathcal{R}<25.5)$ at $z=3$ using SFR-C, in the SCDM cosmology. Bold lines indicate the results of the burst models, and light lines the no-burst models. The histograms are normalized to unit total probability.



Figure 6. Baryonic half-mass radius $r_{1 / 2}$ vs. circular velocity for the SCDM model galaxies at $z=3$. The top panel shows the no-burst models and the bottom panel shows the models with starbursts. Open circles show bright galaxies $(\mathcal{R}<25.5)$, and small dots show galaxies fainter than this limit (down to $I_{814} \sim$ 28). The horizontal line shows the average half-light radius of the observed LBGs in the HDF Giavalisco, Steidel, \& Macchetto 1996; Lowenthal et al. 1997).

local galaxies, as we showed in SP98. Here the significance of this comparison is less clear, since the half-light radius in the UV could be considerably smaller than the baryonic half-mass radius that we model, so we should not necessarily expect these quantities to agree. Although, the K-band radii from new NICMOS observations are similar to the I-band sizes used here (M. Pettini, private communication).

The velocity dispersions of the observed LBGs can be estimated based on the widths of emission lines such as $\mathrm{H} \beta$ 


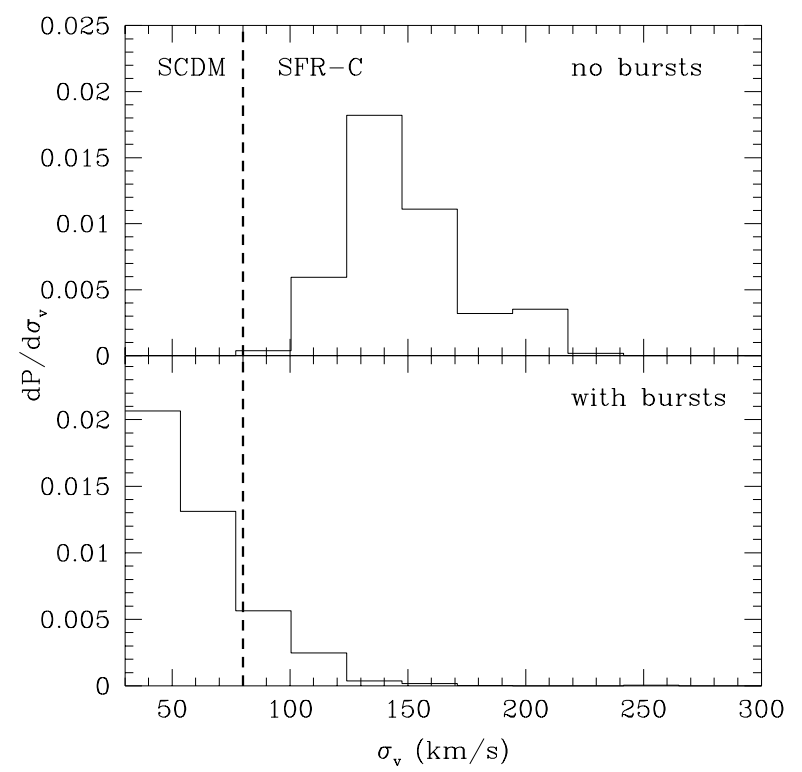

Figure 7. The distribution of 1-D internal velocity dispersions of "LBGs" $(\mathcal{R}<25.5)$ at $z=3$ for SCDM. The top panel shows the no-burst models, and the bottom panel shows the models with starbursts. The vertical line shows the average velocity dispersion of the observed LBGs derived from the line-widths of $\mathrm{H} \beta$ and O[III] emission lines (Pettini et al. 1998)

or $\mathrm{O}[\mathrm{III}]$. Emission lines have been detected for a few of the brightest LBGs from the ground. Velocity dispersions derived from the observed line-widths are $70-90 \mathrm{~km} \mathrm{~s}^{-1}$ for four objects, $50 \mathrm{~km} \mathrm{~s}^{-1}$ for one object, and $150 \mathrm{~km} \mathrm{~s}^{-1}$ for one object (Pettini et al. 1998). The widths of the observed emission lines probably reflect the mass within about one effective radius. At such small radii, there is probably little contribution from dark matter. We therefore estimate the $1-\mathrm{D}$ velocity dispersion in our models using the expression

$\sigma^{2}=\frac{G m}{c r_{e}}$

where $m$ is the total baryonic mass (cold gas and stars), $r_{e}$ is the effective radius (we use the baryonic half-mass radius), and and $c$ is a geometry dependent factor (cf. Phillips et al. 1997), expected to be $c \simeq 5-6$, corresponding to a hot component with a density $\rho \propto r^{-\alpha}$ where $\alpha=2.5-3$ (cf. Binney \& Tremaine (1987)). In Fig. 月, we have used $c=5$, corresponding to $\alpha=2.5$, but note that the results would decrease by about 10 percent for $\alpha=3$.

If the mean velocity dispersion of the very small sample with measured line-widths is representative of the population as a whole, then the velocity dispersions resulting from the baryonic mass alone (no dark matter) in the no-burst models appear to be too large. These models predict that observation of a velocity dispersion smaller than $100 \mathrm{~km} \mathrm{~s}^{-1}$ is unlikely. In addition, because of the difficulty of the observations, line-widths have been obtained for the brightest objects. In the no-burst models, the luminosity is strongly correlated with the stellar mass and hence with the velocity dispersion. Had we instead selected objects brighter than $\mathcal{R}=24.5$, we would have found velocity dispersions in excess of $200 \mathrm{~km} \mathrm{~s}^{-1}$. In the burst models, the probability distribu-

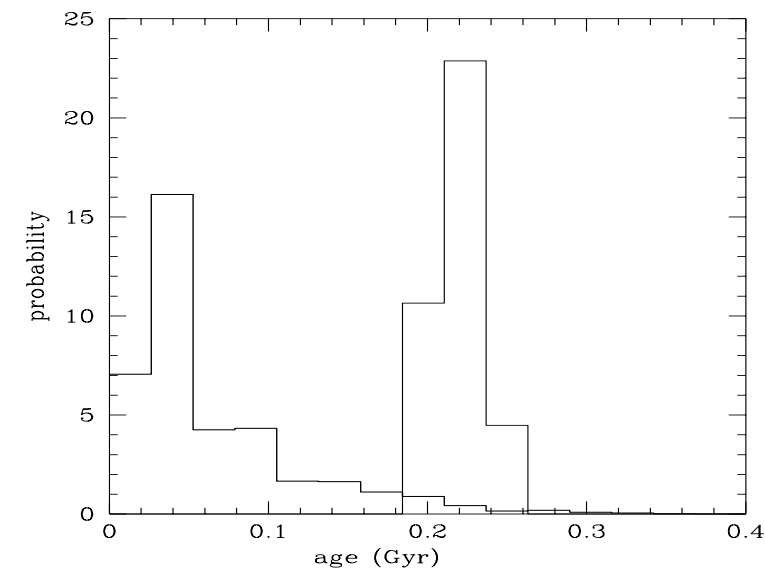

Figure 8. The distribution of luminosity weighted ages for "LBGs" (galaxies brighter than $\mathcal{R}=25.5$ ) at $z=3$ identified in the SCDM models with (bold histogram) and without (light histogram) bursts.

tion for the velocity dispersion peaks at about $65 \mathrm{~km} \mathrm{~s}^{-1}$ and extends up to about $150 \mathrm{~km} \mathrm{~s}^{-1}$. Selecting brighter galaxies $(\mathcal{R} \lesssim 24.5)$ shifts the mean up to about $100 \mathrm{~km} \mathrm{~s}^{-1}$. As we shall see, however, the distribution of velocity dispersions is fairly sensitive to the recipe used for quiescent star formation. Because of this and other uncertainties in the modelling, and the very small number of observations, it is difficult to draw strong conclusions on the basis of the velocity dispersions at this time. However, it is clearly an interesting avenue to pursue.

\subsection{Ages and Colours}

There has been some debate about the likely ages of the observed LBGs. The determination of ages from the observed colours is complicated by the degenerate effect of reddening due to dust. Conversely, determining the amount of dust extinction based on the observed spectra is sensitive to the assumed age of the stellar population. Some of the observational papers discussing the LBG population have suggested that the ages may be as large as 1 Gyr (Steidel et al. 1996b; Pettini et al. 1997b), based on the $\mathcal{R}-\mathcal{K}$ colours of the objects. However, Sawicki \& Yee (1998) conclude that the dominant stellar population of the LBGs is less than 0.2 Gyr old, with median ages of $\sim 10-36$ Myr. This conclusion was based on their comparison of model SEDs to photometric data from 5 filter bands (VIJHK) spanning the Balmer break. The IR photometric data is helpful in breaking the age-extinction degeneracy, although some degeneracy remains.

Fig. 8 shows the distribution of ages for model galaxies at $z=3$ with $\mathcal{R}<25.5$. The model ages are luminosity weighted in the $\mathrm{H}$ filter band (rest $4120 \AA$ at $z \sim 3$ ), which is just redwards of the Balmer break, to facilitate comparison with the Sawicki \& Yee (1998) results. In the no-burst models, the galaxies that satisfy the magnitude cut span a rather narrow range in ages from approximately 0.19 to 0.26 Gyr. In the burst models, however, by far the majority of the galaxies are less than 0.1 Gyr in age, although few are younger than $20 \mathrm{Myr}$. Note that, since metal pro- 


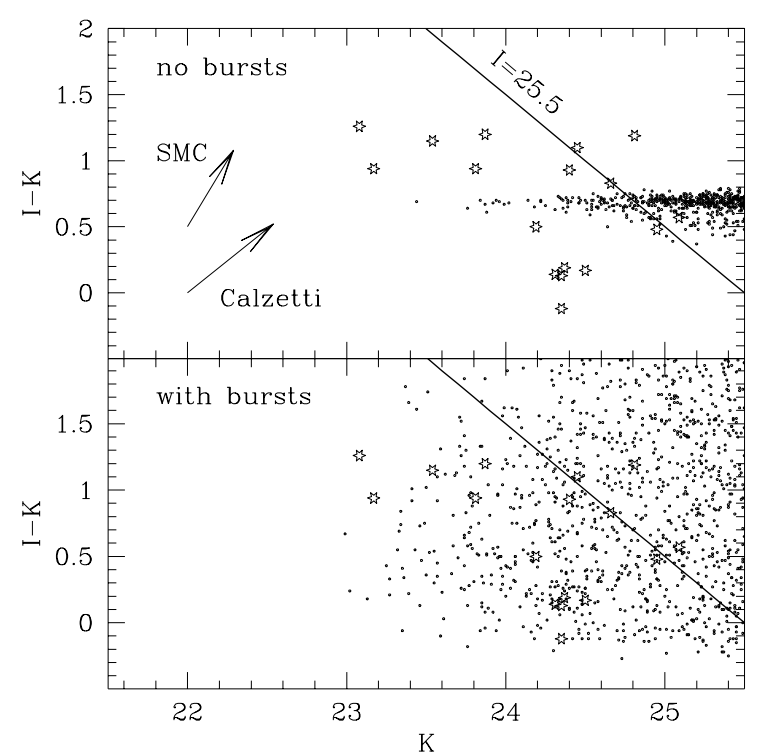

Figure 9. Observed I-K colours for LBGs compared with model predictions at $z=3$. The large star symbols show the $\mathrm{I}_{814}-\mathrm{K}_{\mathrm{s}}$ (AB) colours of LBGs in the HDF with spectroscopically confirmed redshifts $z>2$, as given by Sawicki \& Yee (1998). The small dots show the colours of model galaxies in the no-burst (top panel) and burst (bottom panel) models. Dust extinction would move the model galaxies upwards and to the right, as indicated by the arrows (these assume a factor of 3 extinction in $\mathcal{R}$; see text). Galaxies brighter than $I_{814}=25.5$ lie below the diagonal line.

duction and thus extinction are expected to grow with the time since the onset of the burst, the bright galaxies used in the Sawicki \& Yee (1998) analysis are perhaps being caught in the early part of their star formation episode, which may partly explain why their median ages according to Sawicki \& Yee (1998) are even younger than the burst model estimates in Fig. 8. Also, the Sawicki \& Yee (1998) models assume a single instantaneous burst of star formation or a constant star formation rate, whereas in our models, starbursts occur over a time-scale of $50-100 \mathrm{Myr}$, as expected on the basis of dynamical arguments and as predicted by hydrodynamic simulations. In addition, our model galaxies have more complex (and presumably more realistic) star formation histories. However, on the whole, it is interesting that the ages in both the burst and no-burst models are more consistent with the estimates of Sawicki \& Yee (1998) than with the much larger ages ( $\sim 1$ Gyr associated with the original massive progenitor scenario. As we discuss further in Section 4.3, the ages of the model galaxies are surprisingly insensitive to the assumed cosmology.

We might now wonder whether the colours of the model galaxies are consistent with the observations. In Fig. 9 we show the predicted unreddened $\mathrm{I}-\mathrm{K}$ colours and compare them to the observed colours (approximately rest $\mathrm{U}-\mathrm{V}$ at $z \sim 3$ ) for the models with and without starbursts. We have taken the $\mathrm{K}_{s}$ magnitudes for the LBGs with spectroscopically confirmed redshifts in the HDF from Table 1 of Sawicki \& Yee 1998). We have used the standard Johnson K filter for the models, which has an effective wavelength of $\sim 2.2 \mu$. As usual all magnitudes are in the AB system. The colours

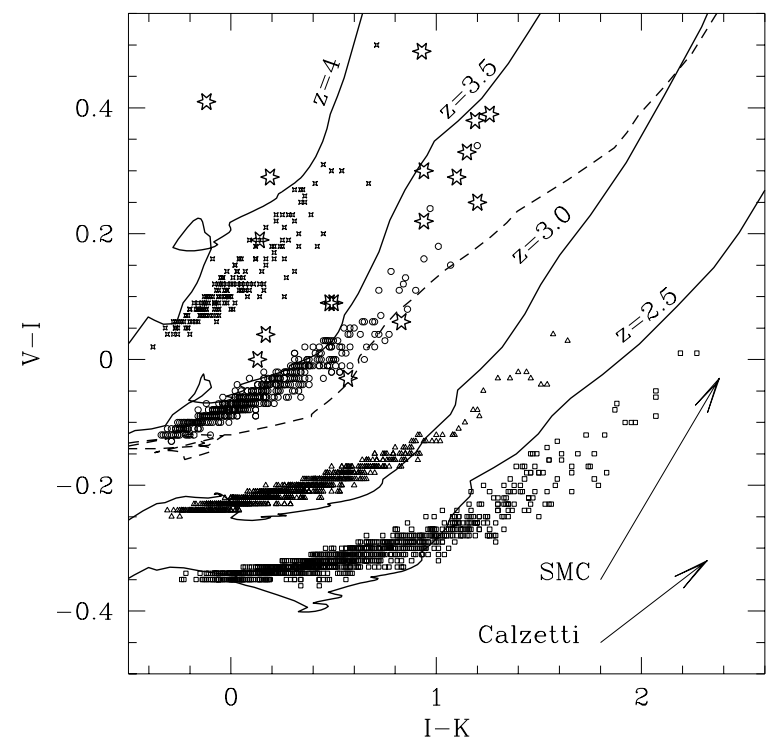

Figure 10. Two-colour diagram (observed V-I vs. I-K) for LBGs. Large star symbols show the $\mathrm{V}_{606}-\mathrm{I}_{814}$ vs. $\mathrm{I}_{814}-\mathrm{K}_{s}(\mathrm{AB})$ colours of LBGs in the HDF with spectroscopically confirmed redshifts $z>2$, as given by Sawicki \& Yee (1998). The small symbols show the unreddened colours of model galaxies with $\mathrm{I}_{814}<25.5$ at $z=2.5$ (squares), $z=3.0$ (triangles), $z=3.5$ (circles) and $z=4$ (crosses), in the burst models. The solid lines show the colours of instantaneous burst populations of various ages from the solar metallicity Bruzual \& Charlot models (GISSEL95), at the same redshifts, as labeled on the plot. The dashed line shows the colours of single burst populations from the multi-metallicity models for $Z=0.2 Z \odot$ (GISSEL96, 1998 updated version) at $z=3$. Most of the observed LBGs are at redshifts of $z \sim 3$ or lower. This suggests the need for dust to reconcile the models with the observed colours. However, note that the low-metallicity models SEDs would require less extinction to bring the colours into agreement with the observations. Dust extinction would move the model galaxies at $z \sim 3$ upwards and to the right, as indicated by the arrows (these assume a factor of 3 extinction in $\mathcal{R}$; see text).

in both the burst and no-burst models are fairly consistent with the observations, but the burst models show a much larger scatter in colour at a given magnitude. In the noburst models, the star formation rate as given by SFR-C is determined solely by the available mass of cold gas, which is tightly correlated with the K magnitude. Note that in order to attempt to draw any conclusions from the scatter in the observed colours, we would need to account for the redshift spread of these photometrically selected galaxies and also for the variable effects of dust. We have also compared the models with the observed $\mathrm{V}_{606}-\mathrm{K}$ colours for the larger sample shown in Dickinson (1998), and find them to be very similar. One might expect the burst models to be bluer than the no-burst models. What we find, however, is that by a redshift of $\sim 3$, many galaxies have experienced several minor merger events with accompanying small starbursts. The older stars from these previous burst events contribute to the rest visual luminosity, leading to a population that is actually brighter in the observed $\mathrm{K}$ band and redder in observed $\mathrm{I}-\mathrm{K}$. We have estimated the effects of dust extinction on the colours by assuming that there is a factor of three 
attenuation in the luminosity at rest $1500 \AA$, as before. We then use the reddening recipe for an SMC type extinction curve and the emperical attenuation law of Calzetti, as given in Figure 2 of Calzetti (1997a), to determine the extinction in the (observed frame) V, I, and K filter bands. In the burst models, the colours of the model galaxies with this sort of dust correction would be in fairly good agreement with the observations.

Fig. 10 shows a two-colour diagram (V-I vs. I-K) for observed LBGs compared to burst model galaxies identified at several redshifts. From this we see that, although the $\mathrm{I}-\mathrm{K}$ colours of the model galaxies at $z=3$ span about the same range as the observations, the $\mathrm{V}-\mathrm{I}$ colours of the model galaxies are about 0.2 to 0.4 magnitudes too blue. This is the case in the no-burst models as well. This is consistent with the commonly acknowledged need for some dust in order to explain the colours of the observed LBGs (Steidel et al. 1996b Lowenthal et al. 1997; Pettini et al. 1997b; Sawicki \& Yee 1998). The solid lines in Fig. 10 show the colours of a single burst population of various ages (age increases with I-K) in the GISSEL95 solar metallicity spectral synthesis models Bruzual \& Charlot 1993). We also show the colours of the new multi-metallicity models (GISSEL96, 1998 updated version) for a metallicity of $0.2 \mathrm{Z}_{\odot}$ (close to the mean metallicity of the "LBGs" in our models). The low metallicity models are about 0.2 mag redder in $\mathrm{V}-\mathrm{I}$ for a given $\mathrm{I}-\mathrm{K}$, which would bring the model colours into better agreement with the observations and thus require less dust extinction. We shall discuss other effects of using the low-metallicity stellar population models in Section 4. Once again, the arrows show the effect dust reddening, assuming a factor of three extinction at $1500 \AA$ and an SMC or Calzetti attenuation law. This appears to be about what would be necessary to bring the model colours into good agreement with the observations, especially with the low-metallicity SEDs. The no-burst models would fare similarly.

\subsection{Environment and Clustering Properties}

The ground-based sample of LBGs with spectroscopic redshifts has been used to study the clustering properties of these objects Steidel et al. 1998; Giavalisco et al. 1998; Adelbetger et al. 1998). An immediately striking property of the redshift distribution in the interval $2.5 \lesssim z \lesssim 3.5$ is the presence of large "spikes", or overdensities, in the number of galaxies per redshift bin. In addition, Giavalisco et al. (1998) have shown that the amplitude of the correlation function of the LBGs at $z=3$ is comparable to that of local galaxies. Analyses based on linear theory (Steidel et al. 1998; Adelberger et al. 1998) and using dissipationless N-body simulations (Jing \& Suto 1998; Bagla 1998; Wechsler et al. 1998) have shown that "spikes" such as those in the observed fields are characteristic of the clustering properties of massive halos, $M \gtrsim 10^{12} h^{-1} M_{\odot}$. The virial velocity dispersion of such a halo at $z=3$ in an Einstein-de Sitter universe is about $\sigma_{v}=220 \mathrm{~km} \mathrm{~s}^{-1}$.

Can this be reconciled with the much smaller velocity dispersions indicated by the observed line-widths? The burst scenario may offer a viable means of reconciling these apparently paradoxical observations. Although a substantial fraction of the bright LBGs identified in the burst models are low-mass galaxies, we find that these galaxies are typ-

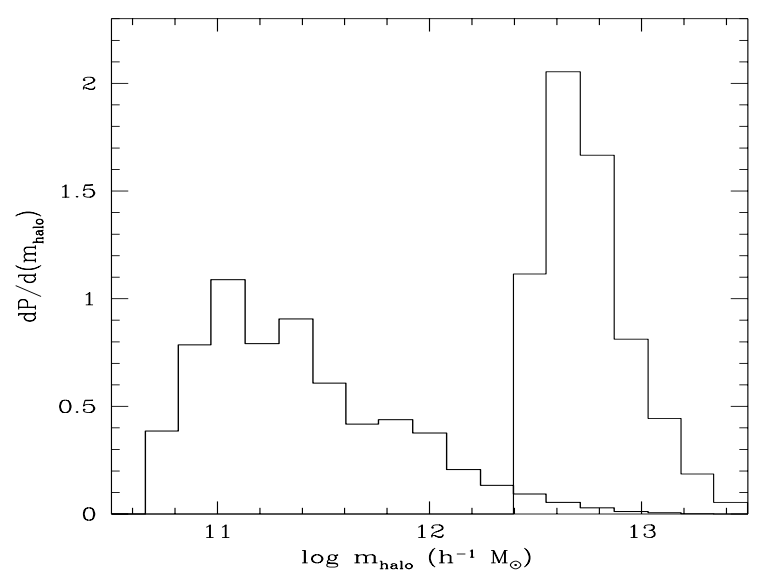

Figure 11. The distribution of the masses of dark matter halos that contain at least one "LBG" (a galaxy brighter than $\mathcal{R}=$ 25.5) at $z=3$, for the no-burst (light histogram) and burst (bold histogram) SCDM models.

ically satellites within relatively massive dark matter halos. This is because smaller halos contain only a few galaxies and thus have low merger rates. In Fig. 11, we show the probability distribution of halo masses for halos containing at least one galaxy with $\mathcal{R}<25.5$ at $z=3$. The "LBG" galaxies identified in the models are contained in halos with $M \gtrsim 2.5 \times 10^{12} h^{-1} M_{\odot}$ in the no-burst models, and $M \gtrsim 10^{11} h^{-1} M_{\odot}$ in the burst models. Wechsler et al. (1998) have shown, using dissipationless N-body simulations, that the "spike" probability and the correlation function for halos with $M \gtrsim 3-9 \times 10^{11} h^{-1} M_{\odot}$ are fairly consistent with the observed quantities for various cosmological models. In the burst models, in addition to lowering the effective mass threshold for halos containing LBGs, the number of LBGs per halo also increases substantially in the most massive halos. We think it is likely that the higher probability of multiple LBGs in the very massive halos found in the "spikes" in the N-body simulations will increase the "spike" probability and steepen the power-law index of the LBG correlation function from -1.5 found in Wechsler et al. (1998) to something closer to the observed value $\sim-1.8$ (Giavalisco et al. 1998). We are currently in the process of performing a more detailed investigation of the clustering properties of "LBGs" in the starburst scenario by combining the results of our semi-analytic models with the N-body simulations analyzed in Wechsler et al. (1998).

If multiple starbursts frequently occur simultaneously in the same halo, we might expect to see a large number of LBGs with small separations $\left(\sim 20-30 h^{-1} \mathrm{kpc}\right.$, which is the physical virial radius of the massive halos at $z \sim 3$ ). This would be in conflict with the observed angular correlation function of LBGs (Giavalisco et al. 1998). Howeverq, it turns out that this is not what the models predict. Because of the characteristic time-scale of the bursts and the rapid decline with mass of the number density of the very large halos that are most likely to contain multiple bursting objects, we find that on average, we would expect to observe two or more LBGs per halo less than 5 percent of the time. This number will be slightly larger for low- $\Omega$ cosmologies, which have higher number densities of massive halos at high redshift. In 
any case, this is not inconsistent with the observed frequency of LBG pairs with $\sim 20-30 \mathrm{~h}^{-1} \mathrm{kpc}$ separations (M. Pettini, private communication; also see Giavalisco et al. 1998). If one looked preferentially in the "spikes", we would predict a much larger number of close pairs, corresponding to multiple bursts per halo. Again, this may be an interesting issue to pursue in more detail using the combined semi-analytic models and N-body simulations.

\subsection{LBGs as Massive Progenitors vs. Bursting Satellites}

Let us summarize what we have learned in the present section. The "Massive Progenitor" scenario Steidel et al. 1996a: Giavalisco, Steidel, \& Macchetto 1996; Steidel et al. 1998; Baugh et al. 1998) supposes that the LBGs are located in the centers of massive dark matter halos, have been forming stars at a moderate rate over a fairly long timescale, and will evolve into the centers of present-day massive ellipticals and spheroids. This is the sort of picture that is supported by our no-burst calculations, or by the semi-analytic modelling work of BCFL98 and Governato et al. (1998). Its predictions are in general agreement with the data (Steidel et al. 1998; Adelberger et al. 1998) regarding the redshift clustering of the LBGs, but we find that its predictions are in disagreement with the observed distribution of the LBGs in redshift and luminosity, and their star formation rates and internal velocity dispersions, especially if even modest dust extinction is taken into account. The luminosity function (Fig. 3) is too steep; in particular, it does not produce enough really bright LBGs. The number of bright LBGs also falls off too rapidly with redshift (Fig. 2). The star formation rates very rarely exceed $10 h^{-2} M_{\odot} \mathrm{yr}^{-1}$ (Fig. [, Fig. 百), and the ages are 200-300 Myr (Fig. 8).

The "Bursting Satellite" scenario that we have introduced here is based on the same sort of semi-analytic modelling that other groups have used (e.g., BCFL98), but we have included the additional physics of the merging of satellite galaxies with each other within larger halos, as well as with the central objects, and the starbursts resulting from these interactions. This modelling simply attempts to capture relevant physical processes that are expected to occur with the hierarchical clustering characteristic of CDM-type structure formation, and it is based on the best available dissipationless and hydrodynamic simulations (Makino \& Hut 1997; Mihos \& Hernquist 1994; Mihos \& Hernquist 1996). Including satellite mergers, the predicted luminosity function at $z \gtrsim 2$ is in significantly better agreement with observations, as a function both of luminosity and redshift. The brightest UV luminosities correspond to star formation rates of $\sim 50 h^{-2} M_{\odot} \mathrm{yr}^{-1}$, but because these are starbursts lasting only several tens of Myr to $100 \mathrm{Myr}$ the total stellar masses are typically $10^{9}-10^{10} h^{-1} M_{\odot}$. The internal velocity dispersions predicted in the Bursting Satellite scenario are significantly lower than those calculated when bursts are neglected (Fig. 7), and in better agreement with the velocity dispersions indicated by the handful of measured linewidths presently available. This suggests that more data on LBG line-widths may be helpful in discriminating between these scenarios. Fortunately, such measurements will become much easier when the new Keck Near-Infrared Spectrograph (NIRSPEC) becomes available, although various biases re- main to be disentangled in modelling and interpreting these line-widths. However, we think that at present the most natural interpretation of the observed line-widths suggests small galaxies whose brightnesses are being amplified temporarily by starbursts.

The predicted colours in the models including starbursts (Fig. 9 and 10) are in good agreement with observations, if corrected for the lower-than-solar metallicity expected and for the presence of dust. Since these starbursts occur mainly in halos massive enough to have significant substructure, the corresponding LBGs are expected to cluster in redshift much as predicted in the Massive Progenitor scenario, in agreement with observations (e.g., Wechsler et al. 1998). Despite the fact that several starbursts are expected to occur in each massive halo as a result of satellite mergers, we find that only in $\lesssim 5 \%$ of cases will multiple LBGs be observed simultaneously in the same halo, which appears to be in agreement with observations.

\section{VARIATIONS}

In the previous section, we considered the properties of galaxies at $z \sim 3$ predicted by semi-analytic models with a certain set of underlying assumptions. We focussed mainly on the differences in models with and without a bursting mode of star formation. In this section, we discuss how the predictions for the luminosity function $\phi(L, z)$ and for stellar masses, sizes, and velocity dispersions of LBGs change if some of the other basic ingredients of the models are varied. In particular, we consider in turn some different recipes for quiescent (non-burst) star formation, several different background cosmologies, a different IMF, and low-metallicity spectral synthesis models.

\subsection{Quiescent Star Formation}

We consider the recipes for treating quiescent star formation and supernovae feedback to be some of the most uncertain ingredients in our models. We now show how our results change if we use the "Durham" package, which has a different treatment of quiescent star formation and supernovae feedback, or the "Santa Cruz 2" package, in which the feedback recipe is the same as in Section 3, but a different recipe for quiescent star formation is used. These packages are described in Section 2.1 and summarized in Table 1. Briefly, we have now introduced a circular velocity or redshift dependence to the star formation efficiency $\tau_{*}$, which previously was assumed to be constant. In this subsection, we continue to use the SCDM cosmology, the Salpeter IMF, and the solar metallicity SEDs as before.

In Fig. 12, we see that changing the recipe for quiescent star formation can have a dramatic effect on the number density of galaxies as a function of redshift. In the "Durham" package, the star formation efficiency is higher for galaxies with higher circular velocity. This leads to much better agreement with the data in the no-burst models, but they still fall short of the observations at bright magnitudes by a factor of 5-10 even with no dust extinction. In the "Santa Cruz 2" package, the star formation efficiency effectively scales as approximately $(1+z)^{3 / 2}$, so quiescent star formation is much more efficient at high redshift. Even without 

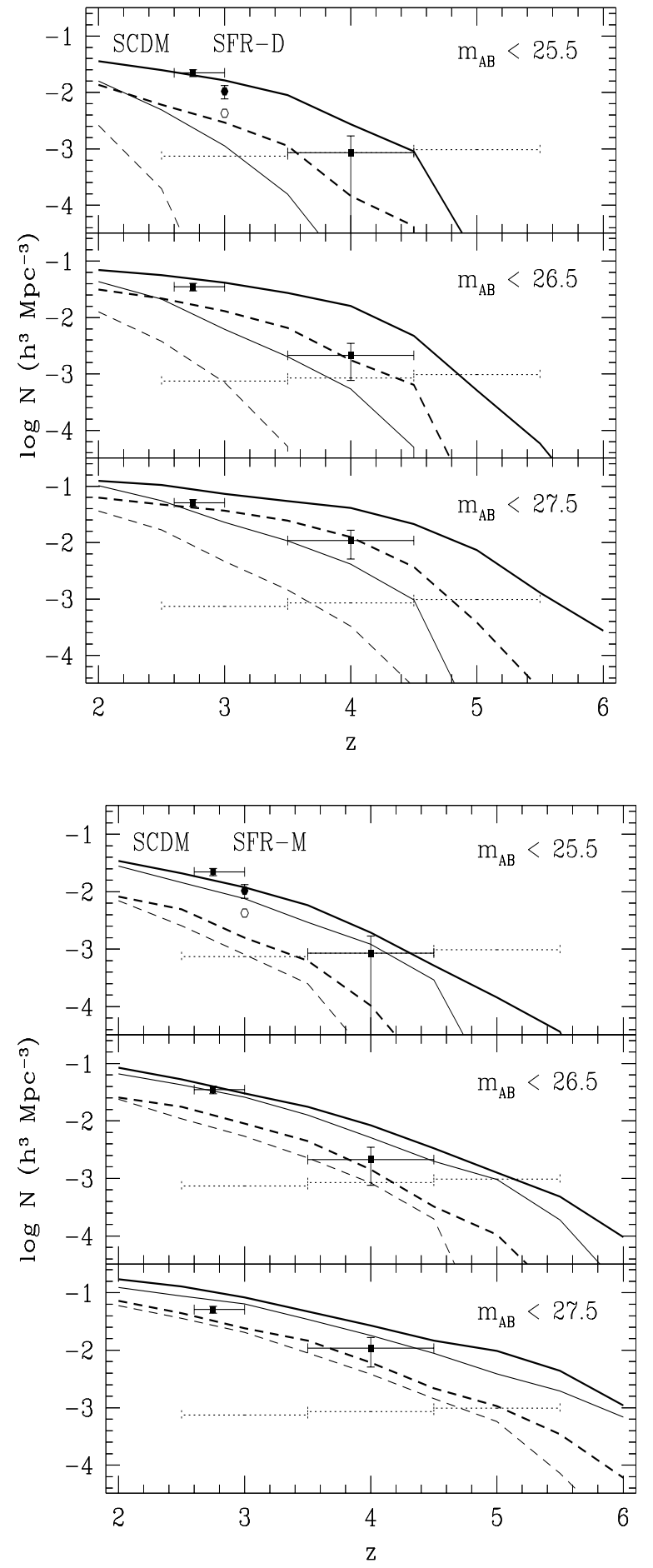

Figure 12. The comoving number density of galaxies, as in Fig. 2, using different recipes for quiescent star formation and supernovae feedback. The top panel shows the "Durham" package (SFR-D), and the bottom panel shows the "Santa Cruz 2" package (SFR$\mathrm{M})$. The efficiency of quiescent star formation is higher at high redshift in SFR-M, leading to more bright galaxies in the no-burst models. starbursts, the models without the dust correction now come very close to matching the observed number density of LBGs in the HDF. Moreover, adding starbursts does not change the number density nearly as much as before. This is because quiescent star formation is now so efficient that it consumes almost all of the available cold gas. Although starbursts occur with the same frequency, their effect is small because there is not much cold gas available to fuel them. However, from the luminosity function at $z=3$ (Fig. 13), we see that the no-burst models still do not produce enough very bright galaxies - the luminosity function cuts off too steeply on the bright end. The starbursts provide just enough additional ultra-bright galaxies to make up this deficit - but once again, only if dust extinction is neglected. The faint end slope of the luminosity function in both the burst and no-burst models is a bit steeper than the value derived by Dickinson (1998), but as we have already mentioned, the faint end slope is not very well constrained by the observations because of possible incompleteness.

Comparing the distribution of the masses of halos that contain LBGs shown in Fig. 14 with Fig. 11 clearly illustrates the difference between adding bright galaxies by the starburst mechanism and by increasing the efficiency of the underlying "normal" (quiescent) star formation. The former process skews this distribution towards smaller mass halos, while the latter shifts the distribution, which retains approximately the same shape, towards smaller mass halos.

The average baryonic half-mass radii of the LBGs in the "Santa Cruz 2" models are about a factor of two larger than our previous results. To understand this, we should recall how sizes are modelled (this is described in more detail in Section 2.3 of SP98). We have assumed that each new batch of collapsing gas ends up at a radius that is a fixed fraction of its radius before collapse (i.e., the cooling radius). The hot gas becomes far more enriched with metals because there has been much more star formation at high redshift. Cooling is more efficient for metal rich gas, and therefore the density necessary for the gas to be able to radiate its energy and cool in a given time is smaller and the corresponding "cooling radius" is larger (see Section 2.2 of SP98 for a description of the cooling model). However, as we mentioned in Section 3.4. the half-light radius in the rest UV may well be smaller than the baryonic half-mass radius that we model.

As we see in Fig. 16, the LBG velocity dispersions in the "Durham" package no-burst models are still quite a bit larger than the observations indicate, but the burst models are in reasonably good agreement with the observed linewidths. In the "Santa Cruz 2" models, the high star formation efficiency in small galaxies shifts the distribution of velocity dispersion in the no-burst models into better agreement with the observations.

We find somewhat broader distributions of ages in the "Durham" package, but with qualitatively similar results to the models of the previous section. The burst and no-burst age distributions are similar in the "Santa Cruz 2" (SFR-M) package, both spanning about 0.1 to $0.3 \mathrm{Gyr}$. This is again because the star formation at high redshift is so efficient that there is very little cold gas left to fuel the starbursts, so they have only a minor affect. The colours of the model galaxies with these alternative star formation and feedback recipes are quite similar to the models of Section 3 and so we do not show them again. 

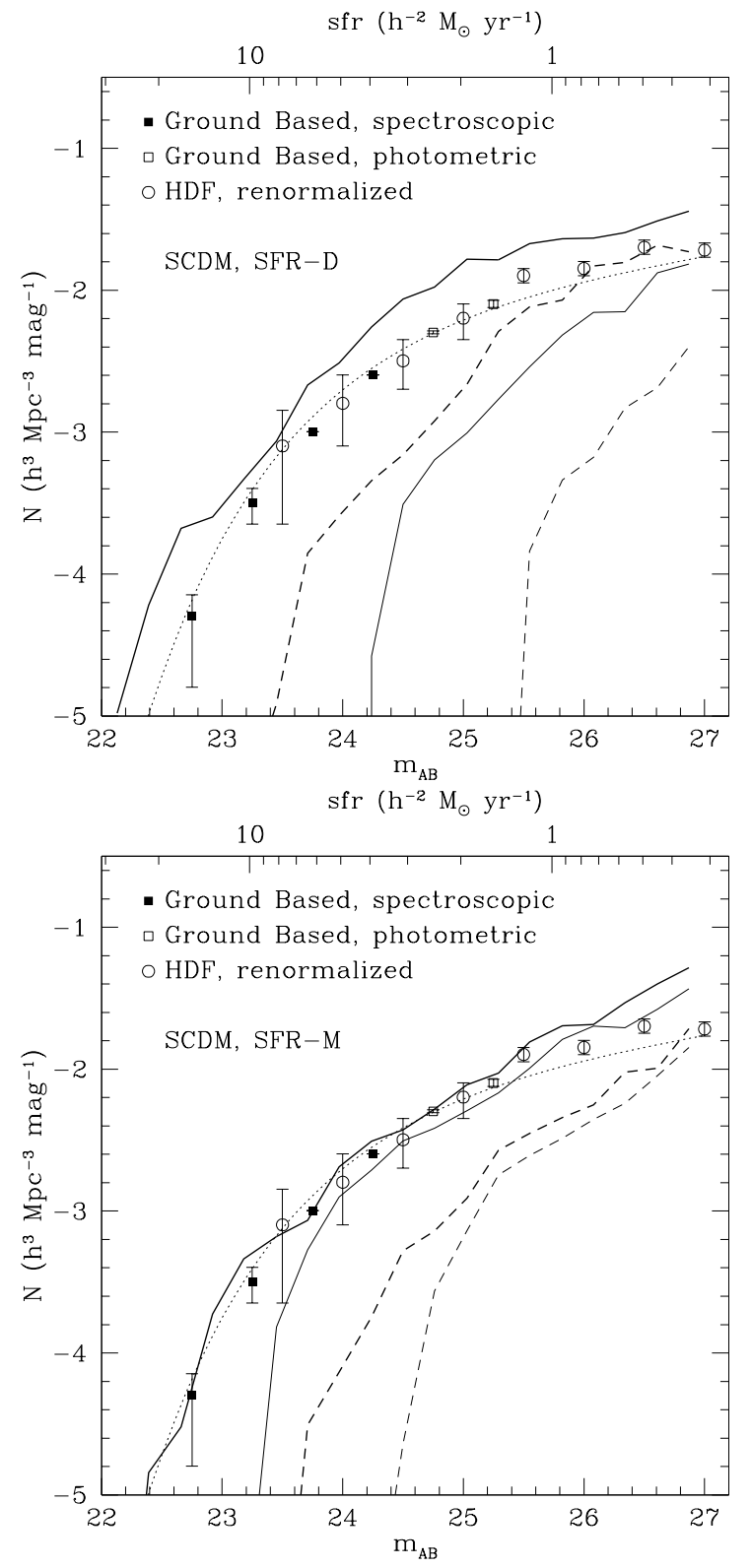

Figure 13. The luminosity function at $z=3$, as in Fig. 3, for two different star formation and supernovae feedback recipes. The top panel shows the "Durham" package (SFR-D), and the bottom panel shows the "Santa Cruz 2" package (SFR-M).

\subsection{IMF and Metallicity Dependence of the SED}

As mentioned earlier, in the previous section we used the Bruzual \& Charlot (1993) GISSEL95 models (which are for solar metallicity stars) with a standard Salpeter IMF to construct the model galaxy spectra. However, as explained at the end of Section 2.1. we set the free parameters by requiring a fiducial reference galaxy with $V_{c}=220 \mathrm{~km} \mathrm{~s}^{-1}$ to have a given luminosity in the I-band at $z=0$. The amount of UV flux for a given I-band flux is quite sensitive to the slope of the IMF (specifically, the fraction of high mass compared to low mass stars) and also depends on the metallicity of the stars. Because most of the $z \geq 2$ observations are in the rest UV, we should investigate the sensitivity of our results to these factors. In Fig. 18, we show the comoving number

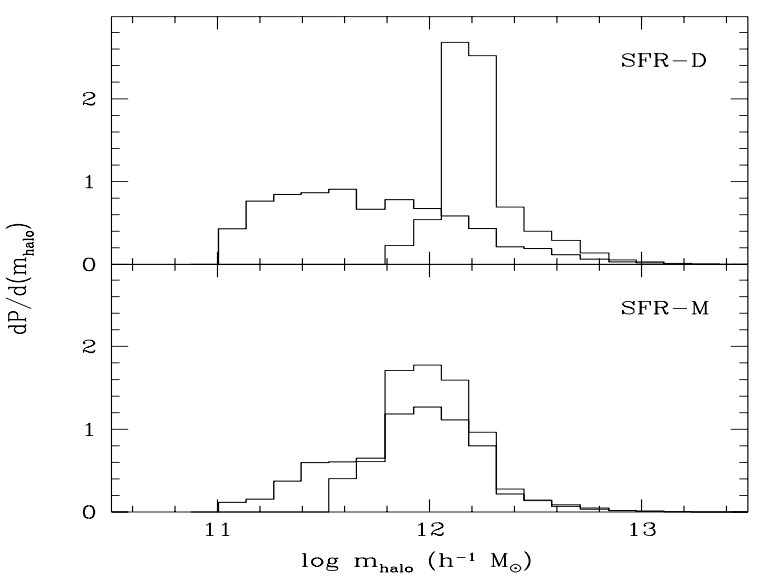

Figure 14. The distribution of the masses of dark matter halos that contain at least one "LBG" (a galaxy brighter than $\mathcal{R}=25.5$ ) at $z=3$, as in Fig. 11, for different star formation and supernovae feedback recipes. The top panel shows the "Durham" package (SFR-D), and the bottom panel shows the "Santa Cruz 2" package (SFR-M). Light and bold lines show the models without and with starbursts, respectively. In the "Santa Cruz 2" package, the distribution is shifted to lower mass halos because SFR-M quiescent star formation is more efficient at high redshift.

density of LBGs for the solar metallicity models with a Scalo IMF (Scalo 1986), and with a Salpeter IMF but using the multi-metallicity GISSEL96 (updated 1998 version) models. We show the results using the SEDs for $0.2 Z_{\odot}$ and $0.02 Z_{\odot}$ stars. We have used the "Durham" package for this calculation. As one can see, the results of the models are quite sensitive to the IMF (as also noted by BCFL98), and less sensitive to the metallicity of the model SEDs. Current observational evidence is consistent with a universal Salpeter IMF; however, this should be considered a large source of uncertainty in any theoretical interpretation of the observations (cf. Scalo 1998). As discussed in the previous section, the no-burst models fall far short of predicting the observed numbers of bright LBGs with even a relatively modest factor of three dust extinction correction, and even the burst models fall somewhat short. Using the sub-solar-metallicity SEDs, which have more flux in the UV relative to the visual part of the spectrum, will help to offset the inclusion of the effects of dust extinction somewhat. However, a somewhat top-heavy IMF, with a larger fraction of high-mass stars, may be needed, especially if the dust extinction corrections are greater than a factor of three. There are indications that such variations in the IMF may be expected in starbursts (Padoan, Nordlund, \& Jones 1997; Chiosi et al. 1997).

\subsection{Cosmology and Power Spectrum}

The SCDM model used in the previous section is generally considered unrealistic; the standard value of $\sigma_{8}=0.67$ is not only drastically incompatible with the COBE normalization (e.g., Górski et al. (1996) give $\sigma_{8}=1.2$ ), it is also marginally incompatible with the cluster abundance at $z=0$, which requires $\sigma_{8} \simeq 0.5-0.6$ for $\Omega=1$ Eke, Cole, \& Frenk 1996; Girardi et al. 1998). The number density of rare objects (such as the massive halos that harbour the LBGs in our models) is very sensitive to the value of $\sigma_{8}$. In the Press-Schechter 

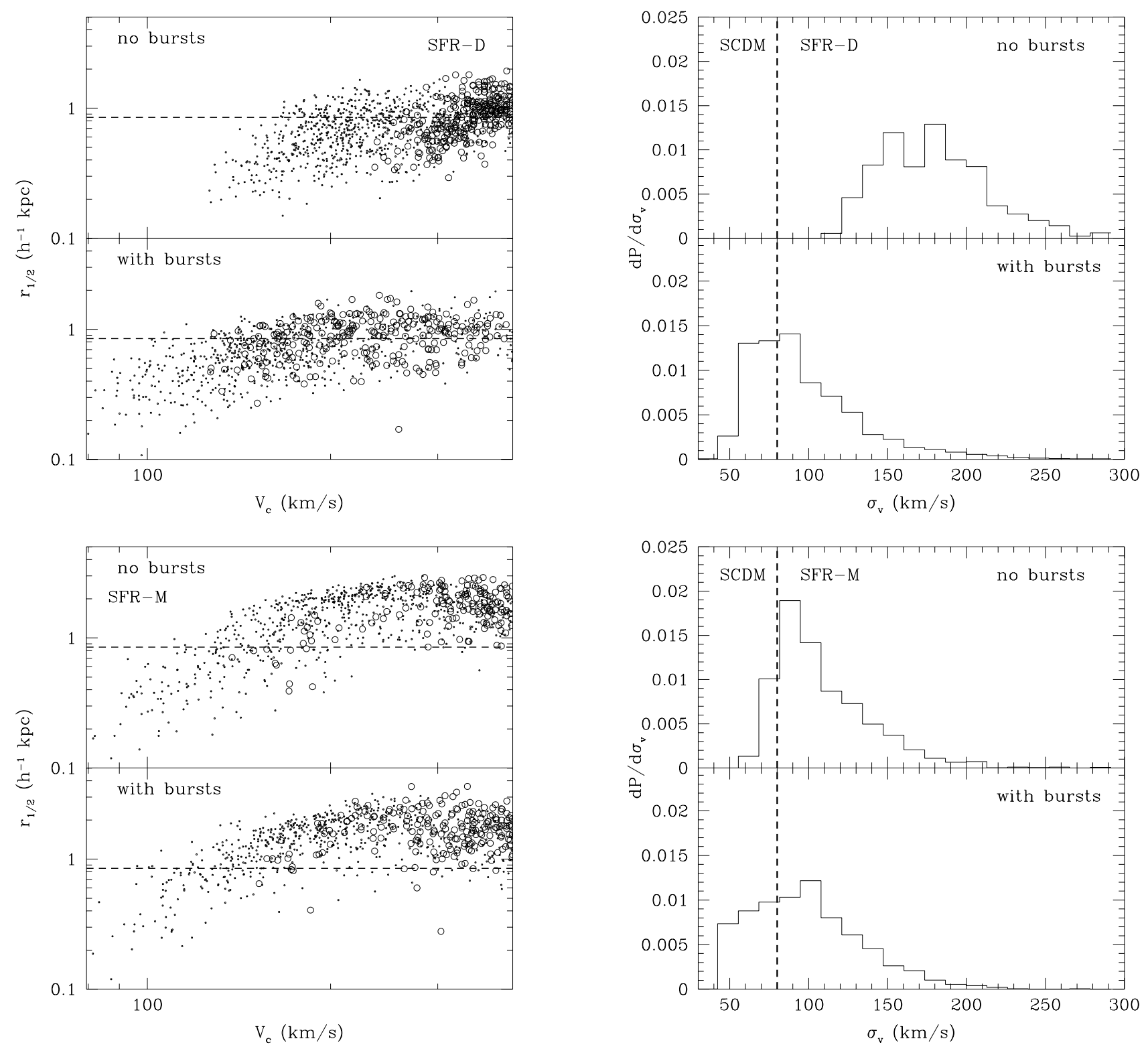

Figure 15. Baryonic half-mass radius $r_{1 / 2}$ vs. circular velocity for the model galaxies at $z \sim 3$ (SCDM). The top panel shows the "Durham" package (SFR-D), and the bottom panel shows the "Santa Cruz 2" package (SFR-M). Open circles show bright galaxies $(\mathcal{R}<25.5)$, and small dots show galaxies fainter than this limit (down to $I_{814} \sim 28$ ). The horizontal line shows the average half-light radius of the observed LBGs in the HDF (Giavalisco, Steidel, \& Macchetto 1996; Lowenthal et al. 1997).

model used here, the halo multiplicity function has an exponential dependence on $\sigma_{8}$, and also depends on the shape of the porfer spectrum through the mass variance $\sigma^{2}(M)$. Its evolution with redshift depends (relatively weakly) on $\Omega_{0}$ and $\Omega_{\Lambda}$ through the linear growth function $D_{\operatorname{lin}}(z)$. All of these factor may potentially effect our results.

We have chosen two of the cosmological models presented in SP98 to illustrate how our results change for different choices of cosmology. The $\Lambda$ CDM model is the same cosmology as Model G of BCFL98, except that our baryon fraction is slightly higher $\left(\Omega_{b}=0.069\right.$ instead of 0.04$)$. The

Figure 16. The distribution of velocity dispersions of LBGs $(\mathcal{R}<25.5)$ at $z=3$, as in Fig. 7) using different recipes for star formation and feedback. The top panel shows the "Durham" package (SFR-D), and the bottom panel shows the "Santa Cruz 2" package (SFR-M).

models are summarized in Table 2, and we refer to SP98 for a more detailed discussion. We do not show results for $\Omega=1$ models with reduced power on small scales (such as "cold plus hot" (CHDM) or "tilted" (TCDM) models (cf. Gross et al. 1998) or the $\tau$ CDM model (cf. Jenkins et al. 1997), despite the fact that these models predict galaxy properties in the nearby universe that are in better agreement with observations than SCDM (cf. SP98), because we find that these models seriously underpredict the comoving number density of LBGs. (Though it should be noted that technical difficulties in treating the two-component CHDM model by the extended Press-Schechter method which is the basis of the semi-analytic calculations reported here make the pre- 

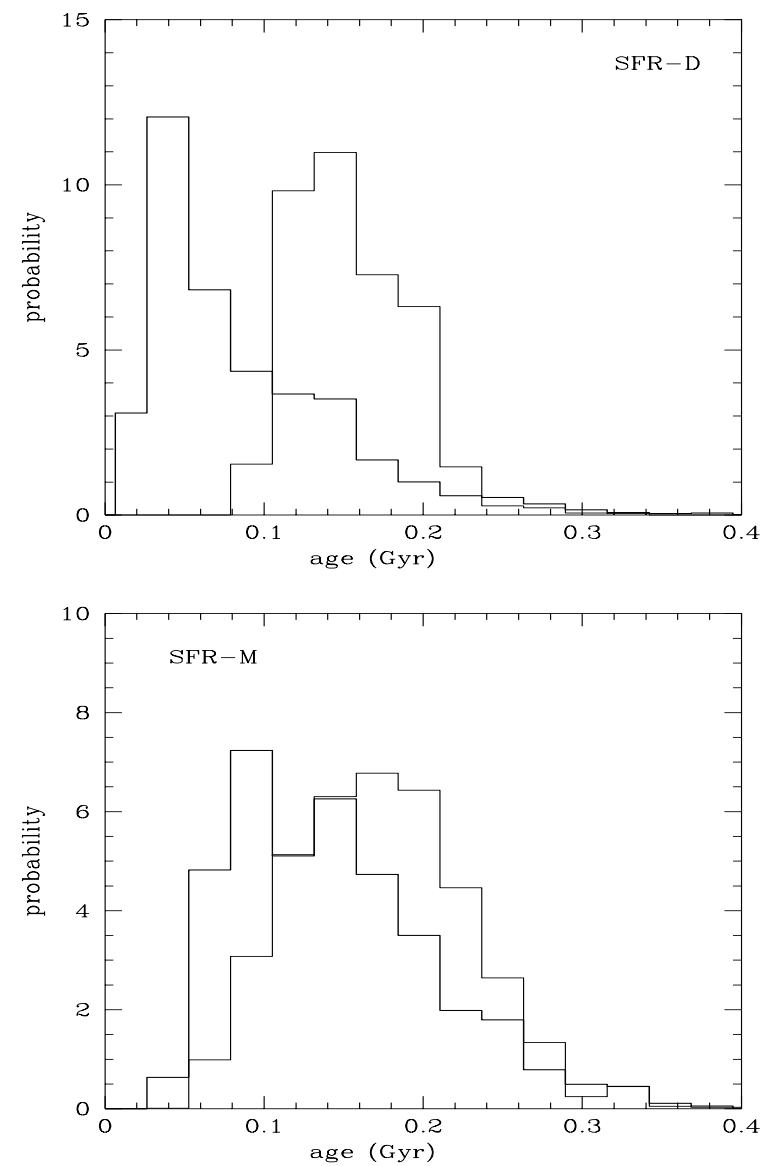

Figure 17. The distribution of luminosity weighted ages for "LBGs" (galaxies brighter than $\mathcal{R}=25.5$ ) at $z=3$ identified in the models with (bold histogram) and without (light histogram) starbursts, for the SCDM cosmology. The top panel shows the "Durham" package (SFR-D), and the bottom panel shows the "Santa Cruz 2" package (SFR-M).

Table 2. Parameters of Cosmological Models. From left to right, the tabulated quantities are: the matter density and density in the form of a cosmological constant in units of the critical density, the Hubble parameter, the age of the universe in Gyr, the bolometric distance modulus at $z=3$, the baryon density in units of the critical density, and the linear rms mass variance on a scale of $8 h^{-1}$ Mpc.

\begin{tabular}{llllllll}
\hline Model & $\Omega_{0}$ & $\Omega_{\Lambda}$ & $h$ & $t_{0}$ & $\mu$ & $\Omega_{b}$ & $\sigma_{8}$ \\
\hline SCDM & 1.0 & 0.0 & 0.5 & 13.0 & 46.9 & 0.1 & 0.67 \\
OCDM & 0.5 & 0.0 & 0.6 & 12.3 & 47.0 & 0.069 & 0.86 \\
$\Lambda$ CDM & 0.3 & 0.7 & 0.6 & 15.7 & 47.3 & 0.069 & 0.97 \\
\hline
\end{tabular}

dicted shortfall in the number density of LBGs less secure for that model). Similarly, we find that models with lower values of $\Omega_{0}<0.3$ significantly underpredict the number of LBGs; in SP98, we showed that such models also underpredict the number of moderately bright galaxies at $z=0$.

Fig. 19 (top panel) shows the comoving number density of galaxies for the $\Lambda$ CDM model, using the "Durham" star formation/feedback package. The no-burst model underpre-

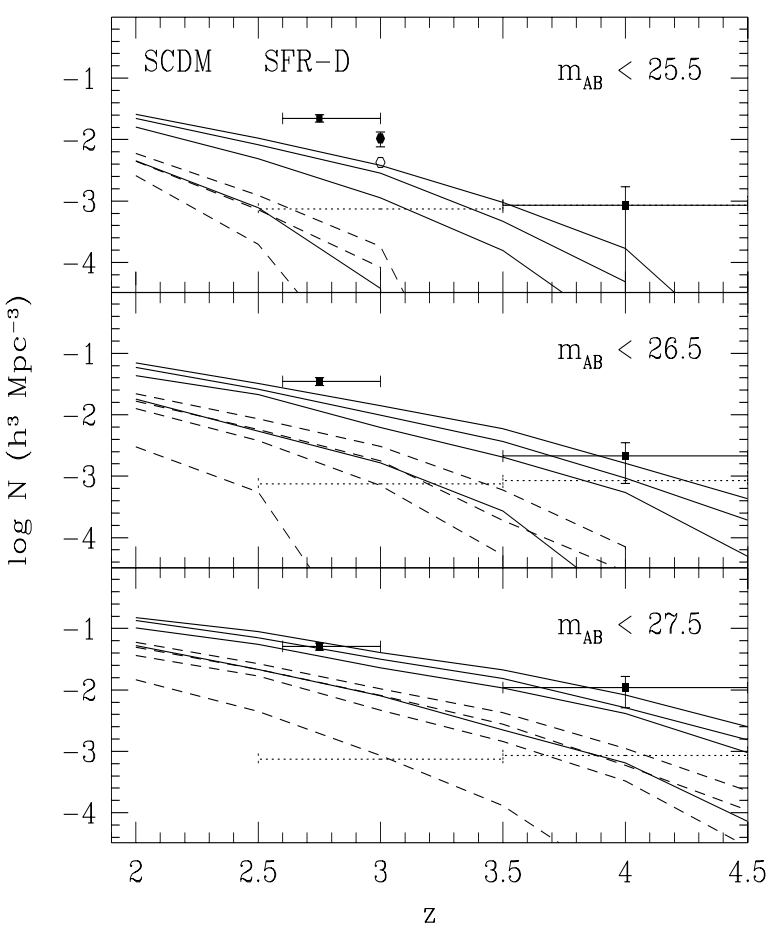

Figure 18. Comoving number densities of LBGs, as in Fig. 2 showing the effects of using low-metallicity model SEDs or changing the IMF. The models shown are all no-burst models with (from highest to lowest, respectively): $Z=0.02 Z_{\odot}$ SEDs, $Z=$ $0.2 Z_{\odot}$ SEDs from the new multi-metallicity GISSELL96 (1998 version) Bruzual \& Charlot models, the original $Z_{\odot}$ model repeated from Fig. 12 (upper panel), and the $Z_{\odot}$ model with a Scalo IMF. Dashed lines show the effects of a factor of 3 dust extinction correction, as in Fig. 2 .

dicts the number density of galaxies by an order of magnitude or more even without dust. Only the burst model with no correction for dust comes close to reproducing the observed number of LBGs, and even this model falls a bit short. The fact that the predicted shortfall of LBGs compared to the data is larger for $\Lambda$ CDM than SCDM might seem counter-intuitive, as one normally expects "earlier structure formation" in a low- $\Omega$ model. However, as we can see from Fig. 20, this rather vague statement can be misleading, as it depends on the mass scale and redshift one is talking about. The low- $\Omega$ models do have larger abundances of cluster mass objects $\left(M \gtrsim 10^{14} h^{-1} M_{\odot}\right)$ at high redshift than SCDM. However, the abundance of smaller mass objects, which dominate the cumulative number density, is lower in this $\Lambda$ CDM model than in SCDM (see Fig. 20). In addition, the luminosity distance at a given redshift is considerably larger in $\Lambda \neq 0$ models (see Table 2 ). Therefore galaxies with larger intrinsic luminosities are selected by the apparent magnitude limit $m=25.5$, and this results in an effective mass cutoff for the halos harbouring LBGs that is about a factor of two larger than in the SCDM models, for both the burst and no-burst models. For the same reason, the ages of LBGs in the $\Lambda$ CDM models are very similar to those found in the SCDM models, even though the universe is $2.7 \mathrm{Gyr}$ older. 

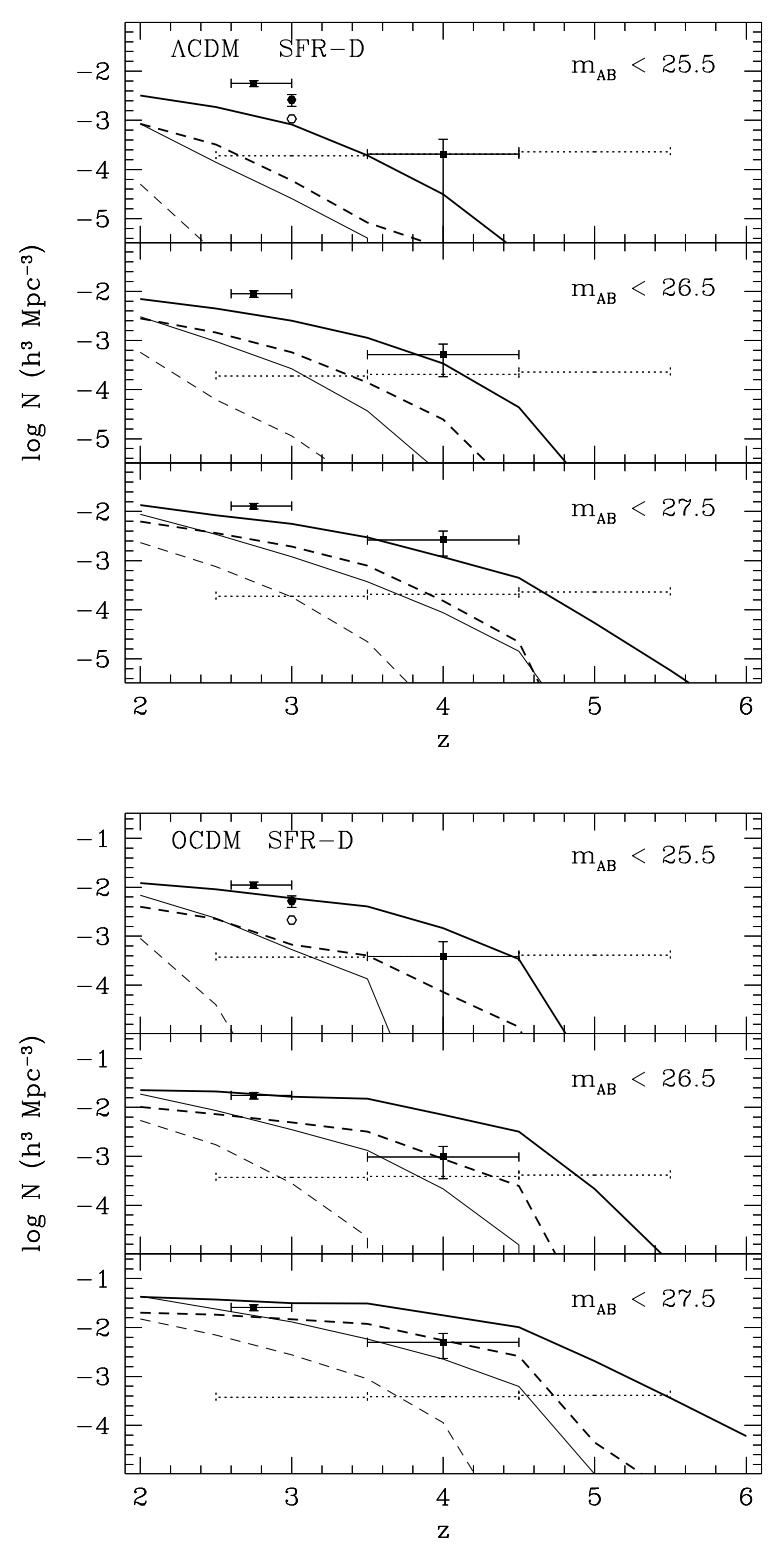

Figure 19. Comoving number densities of observed LBGs, as in Fig. 2, here recalculated for two different cosmologies: $\Lambda \mathrm{CDM}$ (top panel) and OCDM (bottom panel; see Table 2). As before, bold solid lines are for the burst models, light solid lines are for the no-burst models, and dashed lines include a factor of three correction for dust extinction.

The stellar masses of the LBGs identified in the models are also correspondingly larger. As a result, the luminosity function for this model (see Fig. 21, top panel) has a low normalization compared with the observed luminosity function.

In SP98, we showed that the OCDM model with $\Omega_{0}=$ 0.5 produces reasonably good agreement with observations of local galaxies, unlike models with lower $\Omega_{0}$. Comparing Fig. 19 (bottom panel) to Fig. 12 (top panel), we see that the number density of galaxies is a little lower than for SCDM. The luminosity function predicted by the burst models (ignoring dust) at $z=3$ is also consistent with the observed luminosity function of LBGs (Fig. 21, bottom). Thus the

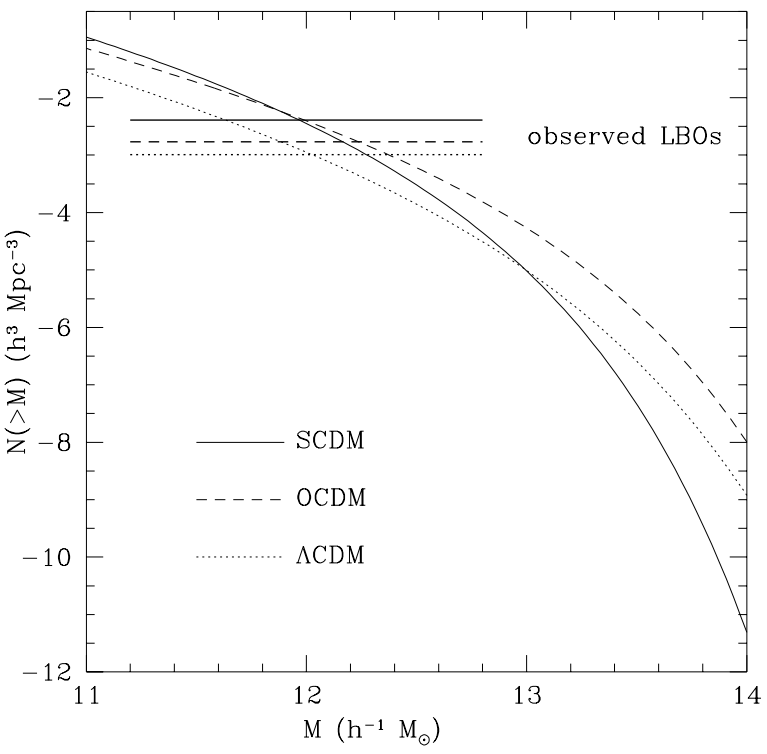

Figure 20. The comoving number density of halos with mass greater than $M$ at $z=3$, from a standard Press-Schechter tophat spherical collapse model. The solid curve is for SCDM, the dashed curve is for OCDM, and the dotted curve is for $\Lambda$ CDM (see Table 2). The horizontal lines show the observed comoving number density of LBGs at $z=3$ from the ground-based sample with spectroscopic redshifts (Giavalisco et al. 1998), computed for the appropriate cosmology.

model predictions compared to the observations are qualitatively similar to what we found using the SCDM cosmology, and bursts are still needed.

\section{GLOBAL QUANTITIES - EVOLUTION WITH REDSHIFT}

\subsection{Star Formation Rate Density}

The compilation of data from local galaxies, the intermediate redshift Canada France Redshift Survey (CFRS; Lilly et al. 1995), and the HDF has made it possible to construct a diagram showing the star formation history of the Universe back to $z=4$ (Fig. 22). This diagram shows an increase in the global star formation rate density of a factor of $\sim 10$ from $z=0$ to $z \sim 1$, a peak between $z=1$ and $z=1.5$, and a shallower decline to a value comparable to the local value by $z=4$. Because of the very large redshift range covered, this compilation consists of star formation rates derived from different indicators. The local star formation rate density $(z=0)$ has been estimated from the H- $\alpha$ survey of Gallego et al. (1995). Recently, the star-formation rate density at $z \sim 0.15$ has also been estimated from the UV survey of Treyer et al. (1997), and from H $\alpha$ measurements of CFRS galaxies (Tresse \& Maddox 1997). Using photometric redshifts, the star formation rates for HDF galaxies in the redshift range $0.5 \lesssim z \lesssim 2$ have been estimated from the UV flux at $\sim 2800 \AA$ (Connolly et al. 1997). At $z=2.75$ and $z=4$, the star formation rate density has been estimated from the UV flux at $\sim 1500 \AA$ for the LBGs in the HDF (Madau et al. 1996; Madau 1996). Some caution 

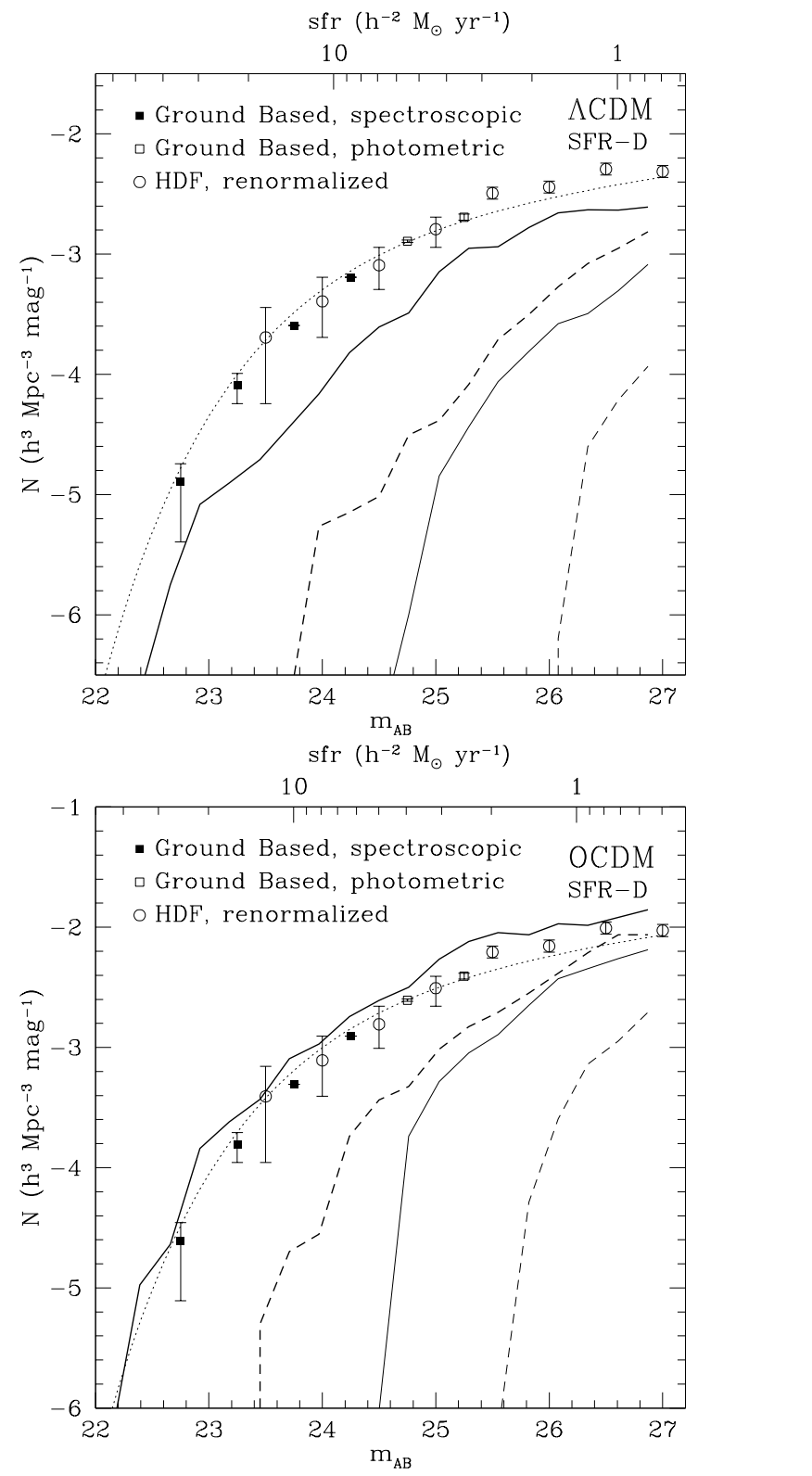

Figure 21. The luminosity function at $z=3$, as in Fig. 3, for the $\Lambda$ CDM (top panel) and OCDM cosmologies. The observed luminosity function from (Dickinson 1998) has been recalculated for these cosmologies.

must be used in interpreting this diagram, as different star formation indicators may have systematic offsets and will be affected to different degrees by dust. One verification of this is the comparison of the star formation rate density $\dot{\rho}_{*}$ at $z \sim 0.15$ from the $2000 \AA$ flux measured by Treyer et al. (1997) with the value of $\dot{\rho}_{*}$ derived from $\mathrm{H} \alpha$ data for CFRS galaxies at the same redshift Tresse \& Maddox 1997). This indicates that the star formation rates derived from the $2000 \AA$ flux must be corrected upwards by at least a factor of $\sim 1.5$. However, the amount of dust presumably also evolves with redshift. Moreover, there is no guarantee that the same population of galaxies is represented in the various samples, which have been selected in very different ways. However, it is encouraging that the star formation rate

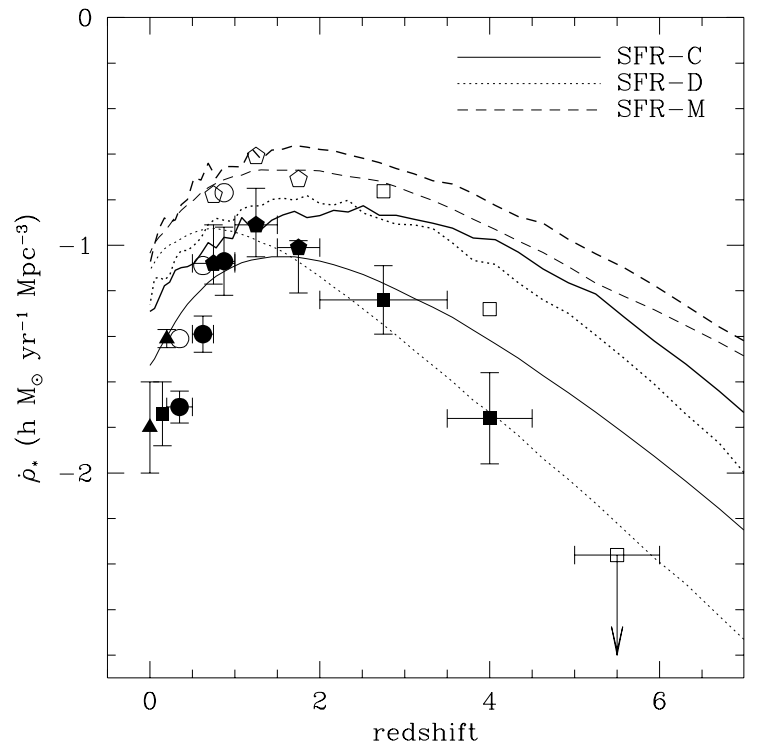

Figure 22. The star formation rate density as a function of redshift. Filled triangles are from $\mathrm{H} \alpha$ abservations of local Gallego et al. 1995) and CFRS (Tresse \& Maddox 1997) galaxies. The filled square at $z \sim 0.15$ is from $2000 \AA$ A fluxes from the UV survey of Treyer et al. (1997). Filled circles are determined from the 2800 $\AA$ fluxes of CFRS galaxies (Lilly et al. 1996), and filled hexagons from the $2800 \AA$ flux of galaxies in the HDF using photometric redshifts Connolly et al. 1997). Filled squares at $z=2.75$ and $z=4$ are from the $\sim 1500$ A fluxes of $U_{-}$and B-dropouts in the HDF (Madau et al. 1996, Madau 1996). Conversions from $\mathrm{H} \alpha$ or UV luminosity are for a Salpeter IMF, as given by Madau, Pozzetti, \& Dickinson (1997). The open symbols show a correction for dust extinction of a factor of two for $2800 \AA$ flux and three for $1500 \AA$ flux, as suggested by Pettini et al. (1997b). The models shown are: Santa Cruz 1 (SFR-C; solid lines), Durham package (SFR-D; dotted lines), and Santa Cruz 2 (SFR-M; dashed lines). Bold and light lines show the models with and without starbursts respectively.

density for the CFRS, which is a magnitude limited survey, is in excellent agreement with the value derived from the photometrically selected sample of Connolly et al. (1997) where they overlap in redshift.

A further uncertainty arises from the unknown slope of the luminosity function at the faint end. All of the observationally derived estimates of the global luminosity density include a correction for incompleteness, obtained by integrating over a Schechter function. The parameters of the Schechter function (particularly the faint end slope) are quite uncertain. In some cases, some of this uncertainly is reflected in the vertical error bars shown on the data points. It should also be noted that various different conversion factors from $\mathrm{H} \alpha$ or UV luminosity to star formation rate are used in the literature. In Fig. 22, we have computed the star formation rate densities from the original flux densities using the appropriate conversion factors for a Salpeter IMF given in Madau, Pozzetti, \& Dickinson (1997).

We show the global star formation rate density as a function of redshift for several variants of the SCDM-based models in Fig. 22. The star formation rate density in the no-burst models with SFR-D and SFR-C decline steeply at 
redshifts greater than $z \sim 2$. This behaviour is very similar to that implied by the observations - if the effects of dust are neglected. If there is a factor of 2 to 3 extinction due to dust, as the most conservative estimates based on the observations indicate, these models actually predict too little star formation at high redshift, as we have already seen. The "Santa Cruz 1" models with SFR-C have a slightly higher star formation rate density at high redshift than the "Durham" package models for two reasons. Star formation is less efficient in small galaxies in SFR-D, and in addition the supernovae feedback in the Durham package is a strong function of circular velocity. In our hierarchical models, halos are smaller on average at high redshift. At low redshift, the star formation rate density is higher in the Durham package models because larger halos, which have more efficient star formation and weaker feedback, are becoming more numerous.

Although the results we obtain for our analogous model are fairly similar to those of BCFL98, we remind the reader that although we have used the same star formation and feedback recipes, our models have been normalized differently from the CAFNZ94 and BCFL98 models and differ in many other details (see SP98). The latter have been normalized to reproduce the observed luminosity function (neglecting extinction) at $z=0$, which is achieved by assuming that 64-67 percent of the stellar mass is in the form of non-luminous brown dwarves, and leads to galaxies that are $\sim 1.8$ magnitudes too faint at a given circular velocity compared to the observed Tully-Fisher relation (CAFNZ94). We normalize our models to match the zero-point of the observed Tully-Fisher relation at $V_{c}=220 \mathrm{~km} \mathrm{~s}^{-1}$ at $z=0$. This requires that all (or at least 90 percent) of the stellar mass is in luminous stars, in which case the Durham package models produce an excess of bright galaxies at $z=0$ compared to the observed luminosity function (SP98). This is also one reason that the star formation rate density at $z \lesssim 1$ in these models is higher than the observations.

In fact, in all the variants of our models, the star formation rate density at $z=0$ is much higher than the Gallego et al. (1995) value (which has been corrected for dust extinction). However, if we consider the observed values of $\dot{\rho}_{*}$ at $z \sim 0.2$ from Tresse \& Maddox (1997), Treyer et al. (1997), and Lilly et al. (1996) with a moderate correction for dust, the models are not so far off. Unless there is extremely strong evolution between $z \sim 0.2$ and $z=0$, this suggests that the Gallego et al. (1995) survey may underestimate the local star formation rate density. Preliminary results from the new KISS survey, which goes to fainter magnitudes and slightly higher redshifts than the Gallego et al. (1995) purvey, indicate that the star formation rate is about a factor of 1.7 times higher than they found (Caryl Gronwall, private communication). In addition, as noted in SP98, the Press-Schechter model used as the basis of our semi-analytic calculation over-predicts the number density of galaxy-sized halos at $z=0$ by about a factor of $1.5-2$. However, the magnitude and mass-scale of this discrepancy evolves with redshift (see Somerville et al. 1998). Correcting for this effect is not straightforward, but will almost certainly also help to improve the agreement of all the models with the observations at $z \sim 0$. It is probably the case, however, that any of the models we have considered so far will have difficulty reproducing the very steep decline in the star formation rate density between $z \sim 0.2$ and $z \sim 1.5$ implied by the CFRS data.

The star formation density in the Durham package and Santa Cruz 1 (SFR-C) models plus starbursts is in good agreement with the observations, if there is about a factor of three correction for dust at $z \sim 2-4$, which is consistent with the dust corrections favored by Pettini et al. (1997b). The evolution of the star formation rate is quite similar in both of these cases, demonstrating that the star formation is dominated by the burst mode and the quiescent star formation mode contributes very little light.

Conversely, the addition of starbursts does not affect the the star formation rate very much in the models with SFR-M. In this case, most of the light is contributed by the quiescent star forming mode. However, in the no-burst models, the results using the different recipes for quiescent star formation (SFR-C, SFR-D and SFR-M) are quite dramatically different. Although $\dot{\rho}_{*}$ is almost the same at $z=0$, the global star formation rate in the no-burst models with SFR$\mathrm{M}$ is almost a factor of 5 higher than the SFR-D no-burst models at $z=3$, and nearly a factor of 20 higher at $z=7$. Such a high $\dot{\rho}_{*}$ at high redshifts is not ruled out by these observations, as it may simply imply a steeper luminosity function than Madau et al. (1996) assumed (cf. Fig. 13) or more extreme dust extinction, although a dust correction that increases with redshift is not what one might expect.

\subsection{Cold Gas Density}

Quasar absorption systems provide an independent means of probing the star formation history of the Universe over a similar redshift range. In particular, observations of damped Lyman- $\alpha$ systems (DLAS) provide an estimate of the $\mathrm{H}_{\mathrm{I}}$ and metal content of the Universe from $z \sim 0.7$ to $z \sim 4$. Fig. 23 compares the models to an estimate of the fraction of the critical density in the form of cold gas for $q_{0}=0.5$ from the observations of Storrie-Lombardi, McMahon, \& Irwin (1996), and for the SCDM models. The observations actually measure only $\mathrm{H}_{\mathrm{I}}$ and contain a correction for helium, but no correction for molecular or ionized hydrogen. The observational estimate shown also contains no correction for dust selection effects, i.e., systems that may have been missed because the background quasars would be too dimmed to be included in an optically selected, magnitude limited sample. Thus the observed values of $\Omega_{\text {cold gas }}$ should be considered lower limits.

Because there is a finite amount of cold, collapsed gas in halos at any given redshift, this quantity is complementary to the luminosity density, which must somehow reflect the mass of cold gas that has been turned into stars. Thus we find that the models with the most inefficient star formation necessarily have the largest amount of cold gas — the Santa Cruz 1 (SFR-C) and Durham package models reproduce the observed density of cold gas at $z \gtrsim 3$, and exceed it at lower redshifts. In order to reconcile these models with the lowredshift data, a large amount of hydrogen must be in forms other than $\mathrm{H}_{\mathrm{I}}$ (note that Young \& Knezek (1989) estimate that the fraction of cold gas in $\mathrm{H}_{2}$ is about 0.5 for spirals, and much smaller for early type galaxies), and/or that many systems were missed due to dust dimming of their background quasars (Pei \& Fall 1995). The model with SFR-D plus bursts has barely enough cold gas at $z>2$ to be consis- 


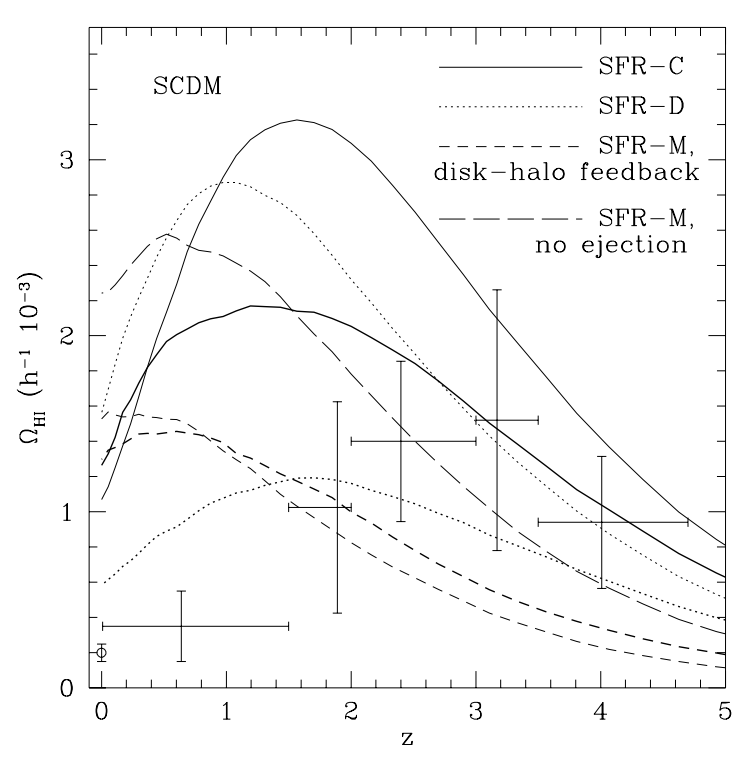

Figure 23. The evolution of $\Omega_{\text {cold gas }}$ as a function of redshift. Data points show the matter density in $\mathrm{H}_{\text {I }}$ from observations of DLAS Storrie-Lombardi, McMahon, \& Irwin 1996). The data point at $z=0$ is from local $\mathrm{H}_{\mathrm{I}}$ observations (Zwaan et al. 1997). The models shown are: Santa Cruz 1 (SFR-C; solid lines), Durham package (SFR-D; dotted lines), Santa Cruz 2 (SFR-M; short dashed lines) and an additional variant in which reheated gas is not allowed to leave the halo (SFR-M; long dashed lines. Note that we have shown only the no-burst version of this model). Bold lines correspond to models including bursts. The quantity plotted for the models is generic "cold gas", some of which may be in the form of molecular or ionized hydrogen, or may be in dust extinguished or low-column density systems that would not be detected as damped systems. Thus the model lines are upper limits on the quantity that is actually determined from the observations $\left(\Omega_{\mathrm{HI}}\right.$ in DLAS) and should lie above the data points.

tent with the observations but manages to skim the bottom of the error bars. At lower redshifts, this model is also consistent with the observations, with a modest correction for contributions from non- $\mathrm{H}_{\mathrm{I}}$ cold gas and dust selection effects, both of which are probably most important at $z<1.5$. The Santa Cruz 1 package (SFR-C) with bursts is also in reasonable agreement with the observations at high and low redshift, though it still has a fairly large excess of cold gas compared to the observations at low redshifts $(z \lesssim 2)$.

The Santa Cruz 2 (SFR-M) package, with and without bursts, predicts substantially less cold gas than the observational estimates at $z \gtrsim 2$. This shows that that the more efficient SFR-M star formation recipe in the Santa Cruz package is using up too much gas at high redshifts. Gas is also ejected from the halos by supernovae in this package. For comparison, we also show the results of a package in which the reheated gas is not allowed to leave the halo (the "Munich" package of SP98). This does increase $\Omega_{\text {cold gas }}$ to a level that is marginally compatible with the observations. The results for $\Omega_{\text {cold gas }}$ from the Munich package at $z \geq 2$ are similar to the results shown in Fig. 12 of Kauffmann (1996). Our models have more cold gas at low redshift, partially because Kauffmann (1996) included only gas in halos with $35 \mathrm{~km} \mathrm{~s}^{-1}<V_{c}<300 \mathrm{~km} \mathrm{~s}^{-1}$, and also perhaps be-

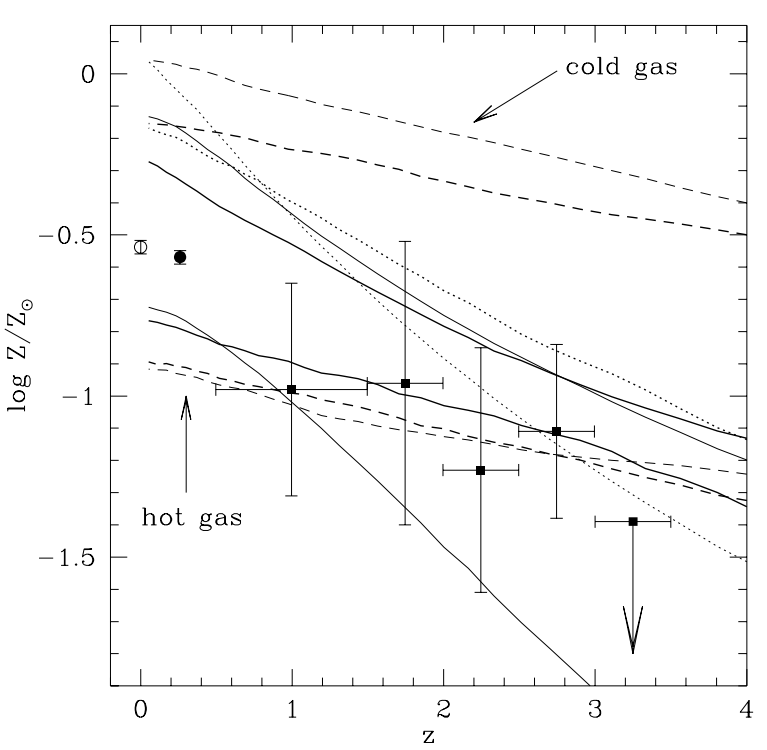

Figure 24. Mean metallicity of cold and hot gas as a function of redshift. The square symbols are from the measurements of Zn abundance in DLAS ([Zn/H $\left.\left./ \mathrm{HLA}_{\mathrm{DL}}\right]\right)$ from Pettini et al. (1997a). The filled dot at $z \sim 0.3$ is from measurements of Fe abundance in hot X-ray gas in clusters (Mushotszky \& Loewenstein 1997), and the open $\operatorname{dot}$ at $z=0$ is the Fe abundance from local clusters Yamashita 1992; Butcher 1995). The models shown are as in Fig. 22. Two sets of lines are shown; the higher abundances reflect the metal content of the cold gas in the models, and the lower abundances the metal content of the hot gas in the models. Note that in the "Durham" package, hot gas always remains at zero metallicity because metals are deposited in the cold gas and all reheated (enriched) gas is ejected from the halo.

cause of the different normalization of the reference galaxy (see SP98). Most of the models have large excesses of cold gas at $z=0$. Our comment in Section 5.1, that the PressSchechter model over-predicts the number density of galaxysized halos at low redshift, but not at higher redshifts, is also relevant here. In addition, the combined effects of dust selection effects and contributions from qhydrogen in forms other than damped systems (molecular and ionized hydrogen or low column density systems) may be considerable.

\subsection{Metals}

The mean metallicity of the DLAS can be estimated from Zn II and Cr II absorption lines. Fig. 24 shows the columndensity weighted average metallicity of DLAS as a function of redshift from Pettini et al. (1997a). We have also shown the Fe abundance of hot X-ray gas in clusters at $z \sim 0.3$ (Mushotszky \& Loewenstein 1997) and $z=0$ (Yamashita 1992; Butcher 1995). We show the model predictions for the average metallicity of the cold and hot gas in several variants of the models. The metallicity of the cold gas predicted by almost all the models is considerably larger than that indicated by the DLAS observations at high redshift. This disagreement is especially pronounced in the models with high-efficiency quiescent star formation at high redshift (SFR-M). The same dust selection effect discussed in the context of the estimates of $\Omega_{\text {cold gas }}$ may lead to an un- 
derestimate of the mean metallicity (because more metal rich systems will also be more dusty and the most likely to be missed). This effect could cause the metal content of the Universe to be underestimated by up to a factor of two or three (M. Pettini, private communication). Given this, the models with SFR-D and SFR-C are in reasonably good agreement with the data. In the models with SFR-M, the cold gas is much more metal rich than the observed DLAS at all redshifts. This problem would be even further exacerbated in the Munich package shown in Fig. 23, because all the metals are retained in the halo, leading to even more metal enrichment at high redshifts.

The metallicity of the hot gas in our models evolves very little between $z \sim 0.3$ and $z=0$, as in the observations. Thus, contrary to the suggestions of Mushotszky \& Loewenstein (1997), these observations are quite consistent with the predictions of hierarchical models with $\Omega=1$ and do not comprise evidence in favor of low $\Omega$. In our models, we find that the metal abundance of the hot gas is systematically larger in large halos, because gas and metal ejection by supernovae is less efficient. In cluster-sized halos, we find that the abundance of the hot gas is about one-third solar, as in the observations. The global abundance shown here is lower because of the large number of small mass halos, which have lower metal abundances in the hot gas component.

The combination of these three observational quantities ( $\dot{\rho}_{*}, \Omega_{\text {cold gas }}$, and the metallicity of the cold gas) provides a fairly strong constraint on the models because the obvious escape routes that one might invoke to reconcile the models with one set of observations will make the agreement with at least one of the others worse. For example, suppose one tried to reconcile the SFR-M models with the data by arguing that there is a large amount of dust at high redshift, which extinguishes the UV flux so that the observed star formation rate is underestimated, and also hides the high metallicity DLAS, causing the mean metallicity to be underestimated as well. However, this will also mean that the observed estimate of $\Omega_{\text {cold gas }}$ will be lower, and we saw that $\Omega_{\text {cold gas }}$ predicted by these models is already too low compared to the observations. Similarly, although the models with SFR-M but no gas ejection (Munich package) have just barely enough cold gas at high redshift to be reconciled with the observed estimates of $\Omega_{\text {cold gas }}$, the predicted metallicities are in even more serious conflict with those derived from the high redshift DLAS.

Still, it should be kept in mind that there are many simplifications and uncertainties involved in this sort of modelling. We would not like to say that any of these scenarios is conclusively ruled out, because there are too many aspects of the important processes, like star formation and supernovae feedback, that are complicated and poorly understood. However, the results we obtain can guide us in deciding which processes, and which details of these processes, are important to understand better or to model in more detail. For example, we have seen that the results are quite sensitive to the ejection of gas and metals from the halos by supernovae feedback. If ejection of metals is considerably more efficient that the ejection of cold gas, as suggested in recent hydrodynamic simulations of dwarf galaxies (Mac Low \& Ferrara 1998 , then the discrepancy with the observed metallicity of the DLAS could be alleviated. Also, we have assumed that in the models with gas ejection, once the gas has left the halo it is never re-accreted. If the gas is re-incorporated into the halo when collapse occurs on larger scales, this could help the problems with $\Omega_{\text {cold gas }}$ (although it will still not help the metal problem, unless some process like the more efficient ejection of metals mentioned above is also invoked). Most likely, comparison with galaxy simulations incorporating supernovae feedback will be necessary in order to better understand these issues.

\section{DISCUSSION}

We have investigated the predictions of semi-analytic hierarchical models for the properties of high-redshift galaxies and the global star formation history of the Universe. We find that the results are particularly sensitive to the treatment of star formation. We have considered three recipes for quiescent star formation and a model for starbursts triggered by galaxy-galaxy interactions.

The galaxy-galaxy interaction rate predicted by simple scaling laws applied within the CDM-based merging hierarchy is considerably higher in the dense halos that collapse at high redshifts. If galaxies are gas rich, this leads to a substantial population of bright starburst galaxies at redshifts $z \gtrsim 2$. At lower redshifts, halos are less dense and galaxies less gas rich, so starbursts are rarer and less dramatic events. Unless quiescent star formation was considerably more efficient in the past, we predict that the majority of the observed LBGs are young, low-mass, starbursting objects. Even in models with very efficient quiescent star formation, a burst mode appears to be necessary to produce as many very bright LBGs as are observed at $z \sim 3$. This is the case in both $\Omega=1$ and low- $\Omega$ cosmologies.

Although the galaxies that are bright enough to be classified as "LBGs" in our models have intrinsic luminosities of about $L_{*}$ or greater, they are relatively young ( 0.1 to 0.3 Gyr), and have small baryonic half-mass radii $\left(\sim 1 h^{-1} \mathrm{kpc}\right)$. This is consistent with the ages for the observed LBGs in the HDF derived by Sawicki \& Yee (1998) based on broad-band SEDs, and with the half-light radii of the observed objects Giavalisco, Steidel, \& Macchetto 1996; Lowenthal et al. 1997). The colours (V-I and I-K) of the model galaxies are consistent with the observations if we include a moderate correction for reddening due to dust (a factor of three extinction at $1500 \AA)$, especially if low-metallicity $\left(Z=0.02 Z_{\odot}\right)$ model SEDs are used. The observed line-widths for a handful of the brightest galaxies (on average $\sim 80 \mathrm{~km} \mathrm{~s}^{-1}$; Pettini et al. 1998 are in good agreement with the internal velocity dispersions we derive in the models including starbursts. The velocity dispersions we calculate for the LBGs in the no-burst models are considerably higher than the observations indicate. However, uncertainties in the modelling and the small sample size make it difficult to draw strong conclusions based on these results.

In our models, the starbursting galaxies identified as LBGs are satellite galaxies bound within massive dark matter halos. Their clustering properties will therefore be characteristic of massive halos $\left(M \geq 10^{11} M_{\odot}\right)$. The analysis of N-body simulations by Wechsler et al. (1998, see also Jing $\&$ Suto 1998) suggests that halos of this mass scale will be strongly clustered in redshift space at $z \sim 3$. Thus the "spikes" discussed by Steidel et al. (1998) and Adelberger 
et al. (1998) and the large correlation amplitude derived by Giavalisco et al. (1998) for the observed LBGs are probably not inconsistent with the starburst scenario. The implications of the starburst model for the clustering properties of these objects are an important subject for further study.

Because the LBGs are selected in the UV, the results of the models are very sensitive to the assumed IMF (primarily to the ratio of the mass in high-mass to low-mass stars). We find that adopting a Scalo IMF instead of a Salpeter IMF leads to a comoving number density of LBGs at $z=3$ that is over an order of magnitude smaller. Similarly, if the true IMF in these objects is more top-heavy than the standard Salpeter, this would substantially increase the number density of bright galaxies and the UV luminosity density predicted by the models. We also investigate using lowmetallicity SEDs, and find that while this makes the galaxies brighter and bluer, the effects are comparatively minor. However, both of these effects (sub-solar metallicity and a top-heavy IMF) could be invoked to increase the number of UV bright galaxies. This appears to be necessary, even in the models with starbursts, if there is significant (even a factor of two to three) extinction by dust.

We have investigated several background cosmologies, normalized to approximately reproduce the observed number density of clusters at $z=0$. In cosmologies with very low $\Omega(\Omega \sim 0.2)$, and in $\Omega=1$ models with reduced power on small scales (such as CHDM, TCDM, or $\tau$ CDM), our models substantially underproduce LBGs compared to the observations. The comoving number density of LBGs in a flat $\Lambda \mathrm{CDM}$ model with $\Omega_{0}=0.3$ is also lower than the observations, even if we include starbursts and neglect dust extinction. An open model with $\Omega=0.5$ produces qualitatively similar results to the SCDM model. We conclude that CDM cosmologies with $\Omega \sim 0.4-0.5$ and $h \sim 60-65$, with or without a cosmological constant, are favored by this comparison. Within this window of parameter space, which is favored by many independent observations, the uncertainties due to astrophysical processes (star formation, supernovae feedback, dust, the IMF, etc) are much larger than those associated with the precise values of the cosmological parameters.

The global star formation rate density, the mass density of cold gas, and the metal content of the Universe as a function of redshift are complentary quantities. Taken together, these observations should provide strong constraints on the models. Again we find that our results are very sensitive to the mass and redshift dependence of the star formation efficiency, as well as to the efficiency of gas and metal ejection by supernovae feedback. If we assume that the efficiency of quiescent star formation is higher at high redshift (SFR-M), we produce large enough total number densities of LBGs, but in order to be consistent with the star formation rate density derived from the UV fluxes of LBGs in the HDF (Madau et al. 1996; Madau 1996), these models would require a much larger correction for incompleteness or dust extinction at $z \gtrsim 4$ than is usually favored. The large amount of star formation activity consumes so much cold gas that at $z>2$ the cold gas density $\left(\Omega_{\text {cold gas }}\right)$ is too low compared with the values derived from DLAS observations. In addition, the accompanying metal production leads to much higher metal abundances in the cold gas at $z>2$ than the observed DLAS (Pettini et al. 1997a). Only in the models with relatively inefficient quiescent star formation, and with interaction triggered starbursts, are we able to simultaneously reproduce these three important observations.

The simple recipes used here almost certainly neglect some important aspects of the complicated physics associated with star formation. However, a qualitatively consistent picture of galaxy formation at high redshift seems to be emerging. Let us consider the constraints imposed by the combined observations at $z \gtrsim 2$. We need a population of gas-rich, metal-poor objects (usually not detectable in emission) corresponding to the damped Lyman- $\alpha$ systems, and a population of extremely luminous, actively star-forming, probably sub-solar metallicity objects to explain the LBGs. Thus we need a way to produce enough UV light to account for the luminosity density implied by the LBGs without using up too much cold gas or producing too much metallicity.

Based on observations of local spiral galaxies, Kennicutt (1989) suggested that star formation occurs only if the surface density of the gas exceeds a critical value. This critical surface density, based on the Toomre disk stability criterion (Toomre 1964), is $\Sigma_{c} \propto \kappa \sigma_{\text {gas }}$, where $\kappa$ is the epicyclic frequency and $\sigma_{\text {gas }}$ is the velocity dispersion of the gas. For a flat rotation curve, $\kappa \propto V_{c} / r \propto \tau_{\text {dyn }}^{-1}$, so that the critical density scales as the inverse of the dynamical time of the galaxy. As we have already discussed, the dynamical time is smaller at high redshift because disks are more concentrated and hence smaller for a given circular velocity. Therefore, if we assume that $\sigma_{\text {gas }}$ is constant, the critical density $\Sigma_{c}$ will be higher at higher redshift. Depending on the evolution of the surface density profile of the gaseous disk, it is possible that this will result in quiescent star formation that is either less efficient at high redshift or that has roughly constant efficiency with redshift. This could lead to a population of gas rich disks with low star formation rates and low metallicities (perhaps analogous to the local population of low surface brightness galaxies, cf. Jimenez et al. 1998, that could correspond to the observed DLAS. We have shown that if there are large reservoirs of cold gas available, interactiontriggered starbursts will then comprise a significant fraction of bright galaxies at high redshifts. According to our models, these starburst galaxies could easily account for most of the galaxies identified as LBGs at high redshift. Because they may be experiencing their first significant episode of star formation, their metallicities will probably still be low, and they may be only mildly extinguished by dust.

Another important consideration, neglected here, could be the expected broad distribution of values for the initial halo spin parameter $\lambda$. Higher- $\lambda$ protogalaxies will have larger radii and lower surface densities and hence lower star formation rates - and also larger cross-sections for detection as DLAS (Mo, Mao, \& White 1997). Smaller- $\lambda$ protogalaxies will have higher surface densities, and are more likely to be gravitationally unstable, which according to the Kennicutt condition, is a necessary pre-condition for star formation. The short dynamical time-scales and high surface densities of these objects could lead to a burst-like episode of star formation, even in the absence of any external perturbation due to an interaction. Thus we suggest that these two populations of high-redshift objects, the Damped Lyman- $\alpha$ systems and the Lyman break galaxies, both of which have been identified with "proto-galaxies", may actually have very different properties.

What will be the fate of the satellite-merger starbursts 
in this scenario? Much of the enriched gas is likely to be ejected from these relatively low-mass systems, but be retained within the larger massive halo within which it is a satellite. This may help to explain the large covering factor implied by the high probability with which $\mathrm{C}_{\mathrm{IV}}$ and $\mathrm{Mg}_{\mathrm{II}}$ absorption are seen in QSO spectra whenever a bright galaxy at the appropriate redshift is $\lesssim 30 h^{-1} \mathrm{kpc}$ of the QSO line of sight, which is hard to explain as the result of outflow from a central galaxy (Steidel 1995). The stars and globular clusters produced in the starburst are likely to be stripped and eventually accreted by the central galaxy, forming a Pop II stellar halo such as that of the Milky Way, as proposed by Trager et al. (1997). This scenario resembles the picture of galactic stellar halo formation outlined in the classic paper by Searle \& Zinn (1978), which is supported by much recent evidence (cf. Majewski, Munn, \& Hawley 1996, Carney et al. 1996, Spmmer-Larsen et al. 1997 and references therein).

\section{ACKNOWLEDGEMENTS}

We thank Kurt Adelberger, Daniela Calzetti, Mike Fall, Ken Freeman, Mauro Giavalisco, Max Pettini, Jason Prochaska, Marcin Sawicki, and Chuck Steidel for useful discussions. We thank Carlton Baugh, Shaun Cole, Carlos Frenk, Cedric Lacey, Guinevere Kauffmann, and Simon White for their feedback on earlier versions of this work. We also thank Caryl Gronwall, Andrey Kravtsov, James Lowenthal, Jesus Gallego, Rafael Guzman, Drew Phillips, and Scott Trager for useful discussions and general help. We are grateful to Stephane Charlot for providing us with the updated 1998 version of the GISSEL96 non-solar-metallicity model SEDs. RSS acknowledges support from a GAANN fellowship at UCSC and a University Fellowship from the Hebrew University, Jerusalem. RSS, JRP, and SMF have received support for this project from a NASA ATP grant at UCSC.

\section{REFERENCES}

Adelberger K. et al., 1998, ApJ, in press, astro-ph/9804236

Bagla J., 1998, MNRAS, in press, astro-ph/9707159

Baugh C., Cole S., Frenk C., Lacey C., 1998, ApJ, in press, astroph/9703111; (BCFL98)

Baugh C. M., Cole S., Frenk C. S., 1996, MNRAS, 283, 1361

Binney J., Tremaine S., 1987, Galactic Dynamics. Princeton Univ. Press, Princeton, NJ

Bruzual A., Charlot S., 1993, ApJ, 405, 538

Butcher J., 1995, Ph.D. thesis, Leicester University

Calzetti D., 1997a, in Waller W., ed, The Ultraviolet Universe at Low and High Redshift. Woodbury: AIP Press

Calzetti D., 1997b, AJ, 113, 162

Calzetti D., Kinney A., Storchi-Bergman T., 1994, ApJ, 429, 582

Carney B., Laird J., Latham D., Aguilar L., 1996, AJ, 112, 668

Chiosi C., Bressan A., Portinari L., Tantalo R., 1997, astro$\mathrm{ph} / 9708123$

Cole S., Aragón-Salamanca A., Frenk C., Navarro J., Zepf S., 1994, MNRAS, 271, 781, CAFNZ94

Connolly A., Szalay A., Dickinson M., SubbaRao M., Brunner R., 1997, ApJ, in press, astro-ph/9706255

Dickinson M., 1998, in M. Livio S. F., Madau P., ed, The Hubble Deep Field

Eke V. R., Cole S., Frenk C. S., 1996, MNRAS, 282, 263

Gallego J., Zamorano J., Aragón-Salamanca A., Rego M., 1995, ApJ, 455, L1
Giavalisco M., Steidel C., Adelberger K., Dickinson M., Pettini M., Kellogg M., 1998, ApJ, in press, astro-ph/9802318

Giavalisco M., Steidel C., Macchetto D., 1996, ApJ, 470, 189

Giovanelli R., Haynes M., Salzer J., Wegner G., Da Costa L., Freudling W., 1995, AJ, 110, 1059

Giovanelli R. et al., 1997, ApJ, 477, L1

Girardi M., Borgani S., Giuricin G., Mardirossian F., Mezzetti M., 1998, astro-ph/9804188

Górski K. M., Banday A. J., Bennett C. L., Hinshaw G., Kogut A., Smoot G. F., Wright E. L., 1996, ApJ, 464, L11

Governato F., Baugh C., Cole S., Lacey C., Quinn T., Stadel J., 1998, Nature, 392, 359

Gross M. A. K., Somerville R. S., Primack J. R., Holtzman J., Klypin A. A., 1998, MNRAS, submitted, astro-ph/9712142

Guzman R., Gallego J., Koo D., Phillips A., Lowenthal J., Faber S., Illingworth G., Vogt N., 1997, ApJ, 489, 559

Jenkins A. et al., 1997, ApJ, submitted, astro-ph/9709010

Jimenez R., Padoan P., Matteucci F., Heavens A., 1998, MNRAS, in press, astro-ph/9804049

Jing Y., Suto Y., 1998, ApJ, 494, 5

Kauffmann G., 1996, MNRAS, 281, 475

Kauffmann G., White S., Guiderdoni B., 1993, MNRAS, 264, 201, KWG93

Kennicutt R., 1989, ApJ, 344, 685

Kennicutt R., 1998, ApJ, in press, astro-ph/9712213

Lilly S., Le Fèvre O., Crampton D., Hammer F., Tresse L., 1995, ApJ, 455, 50

Lilly S., Le Févre O., Hammer F., Crampton D., 1996, ApJ, 460, L1

Lowenthal J. et al., 1997, ApJ, 481, 673

Mac Low M.-M., Ferrara A., 1998, ApJ, in press, astro

Madau P., 1996, in Star Formation Near and Far, astro$\mathrm{ph} / 9612157$

Madau P., Ferguson H., Dickinson M., Giavalisco M., Steidel C., Fruchter A., 1996, MNRAS, 283, 1388

Madau P., Pozzetti L., Dickinson M., 1997, ApJ, submitted, astro$\mathrm{ph} / 9708220$

Majewski S., Munn J., Hawley S., 1996, ApJ, 459, L73

Makino J., Hut P., 1997, ApJ, 481, 83

Meurer G., Heckman T., Lehnert M., Leitherer C., Lowenthal J., 1997, AJ, 114, 54

Mihos J., Hernquist L., 1994, ApJ, 425, 13

Mihos J., Hernquist L., 1996, ApJ, 464, 641

Mo H., Mao S., White S., 1997, MNRAS, submitted, astro$\mathrm{ph} / 9707093$

Mushotszky R., Loewenstein M., 1997, ApJ, 481, L63

Padoan P., Nordlund A., Jones B., 1997, MNRAS, 288, 145

Pei Y., Fall S., 1995, ApJ, 454, 69

Pettini M., Kellogg M., Steidel C., Dickinson M., Adelberger K., Giavalisco M., 1998, ApJ, in press

Pettini M., Smith L., King D. L., Hunstead R., 1997a, ApJ, 486, 665

Pettini M., Steidel C., Adelberger K., Kellogg M., Dickinson M., Giavalisco M., 1997b, astro-ph/9708117

Phillips A. et al., 1997, ApJ, 489, 543

Pozzetti L., Madau P., Zamorani G., Ferguson H., Bruzual A., 1998, MNRAS, submitted

Salpeter E., 1955, ApJ, 121, 61

Sawicki M., Yee H., 1998, AJ, in press, astro-ph/9712216

Scalo J., 1986, Fundam. Cosmic Phys., 11, 1

Scalo J., 1998, in G. Gilmore S. R., I. Parry, ed, The Stellar Initial Mass Function, Proceedings of the 38th Herstmonceux Conference. PASP, astro-ph/9712317

Searle L., Zinn R., 1978, ApJ, 225

Somerville R., 1997, Ph.D. thesis, University of California, Santa Cruz, http://www.fiz.huji.ac.il/ rachels/thesis.html

Somerville R., Kolatt T., 1998, MNRAS, in press, astro$\mathrm{ph} / 9711080$ 
Somerville R., Lemson G., Kolatt T., Dekel A., 1998, MNRAS, submitted

Somerville R., Primack J., 1998, MNRAS, in press, astroph/9802268; (SP98)

Sommer-Larsen J. et al., 1997, ApJ, 481, 775

Steidel C., 1995, in Meylan G., ed, QSO Absorption Lines: Proceedings of the ESO Workshop. Springer-Verlag

Steidel C., Adelberger K., Dickinson M., Giavalisco M., Pettini M., Kellogg M., 1998, ApJ, 492, 428

Steidel C., Giavalisco M., Dickinson M., Adelberger K., 1996a, AJ, 112, 352

Steidel C., Giavalisco M., Pettini M., Dickinson M., Adelberger K., 1996b, ApJ, 462, L17

Steidel C., Hamilton D., 1992, AJ, 104, 941

Steidel C., Hamilton D., 1993, AJ, 105, 2017

Storrie-Lombardi L., McMahon R., Irwin M., 1996, MNRAS, $283 \mathrm{~L}, 79$

Toomre A., 1964, ApJ, 139

Trager S. C., Faber S. M., Dressler A., Oemler A., 1997, ApJ, 485,92

Tresse L., Maddox S., 1997, in S. D’Odorico E. G., A. Fontana, ed, The Young Universe. A.S.P. Conf. Series, astro-ph/9712130

Treyer M., Ellis R., Milliard B., Donas J., 1997, in Waller W., ed, The Ultraviolet Universe at Low and High Redshift. Woodbury: AIP Press, astro-ph/9706223

Tully R., Pierce M., Huang J.-S., Saunders W., Verheijen M., Witchalls P., 1998, astro-ph/9802247

Walker I., Mihos J., Hernquist L., 1996, ApJ, 460, 121

Wechsler R., Gross M., Primack J., Blumenthal G., Dekel A., 1998, ApJ, in press, astro-ph/9712141

Williams R. et al., 1996, AJ, 112, 1335

Willick J., Courteau S., Faber S., Burstein D., Dekel A., Kolatt T., 1996, ApJ, 457, 460

Willick J., Courteau S., Faber S., Dekel A., 1995, ApJ, 446, 12

Yamashita K., 1992, in Tanaka Y., Koyama K., ed, Frontiers of X-ray Astronomy. Tokyo: Universal Academy Press

Young J. S., Knezek P., 1989, ApJ, 347, L55

Zwaan M., Briggs F., Sprayberry D., Sorar E., 1997, ApJ, 490, 173 